\title{
GENETICAL AND MOLECULAR STUDIES ON SALINITY AND DROUGHT TOLERANCE IN RICE (ORYZA SATIVA L.)
}

\author{
Farid, M. A. ${ }^{1}$; A. A. Abou Shousha'; M. E. A. Negm² and S. \\ M. Shehata ${ }^{2}$ \\ 1. Department of Genetics, Faculty of Agriculture, Kafrelsheikh \\ University, 33516, Egypt. 2. Rice Research \& Training Center, \\ 33717 Sakha, Kafr EL-Sheikh, Field Crops Research Institute, \\ Agricultural Research Center, Egypt
}

\begin{abstract}
The field experiments were conducted during 2013 and 2014 growing seasons at Kafrelsheikh University and Rice Research and Training Center (RRTC), Sakha, Kafrelsheikh, Egypt, facilities at El-Sirw Agriculture Research Station experimental farm, Damietta governorate, Egypt. Eight-parental half diallel cross and eight SSR molecular markers were used to determine combining ability of some common rice genotypes grown under normal, drought and salinity conditions. Analyses of variance were highly significant for days to heading, plant height, total chlorophyll content, proline content, sodium content, potassium content, $\mathrm{Na}^{+} / \mathrm{K}^{+}$ratio and panicles plant ${ }^{-1}$, filled grains panicle ${ }^{-1}$ and grain yield plant ${ }^{-1}$ indicated highly significant differences among the studied genotypes for this traits. The estimates of GCA effects indicated that three rice genotypes i.e. Giza178, A22 and WAB56-125 were the best general combiners for total chlorophyll content, proline content, sodium content, potassium content, $\mathrm{Na}^{+} / \mathrm{K}^{+}$ratio, panicles plant ${ }^{-1}$, filled grains panicle $^{-1}$ and grain yield plant ${ }^{-1}$ under normal, salinity and drought conditions. The estimates of SCA effects indicated that the most desirable hybrids were Sakha105 x A22, Sakha102 x A22 and Sakha104 x Giza178 for total chlorophyll content, panicles plant ${ }^{-1}$ and grain yield plant ${ }^{-1}$; Giza178 $\times$ WAB56125 for filled grains panicle ${ }^{-1}$; Sakha102 x Sakha104 for days to heading; Sakha104 x Sakha105 for plant height. The genetic distance, measured using SSR markers, differed from 0.00 to 0.79 among the eight genotypes. Microsatellite markers were effective in predicting the mean and the variance of SCA in various cultivar combinations. In addition, RM223 marker elucidated the possibility to use it in MAS for salinity and drought tolerance in the studied rice genotypes according to different alleles.
\end{abstract}

\section{INTRODUCTION}

Rice (Oryza sativa, L.) is one of the most important crops in the world's, providing a staple food for nearly half of the global population (FAO, 2004). In Egypt, rice is considered the second important cereal crop, following wheat, as a main food for Egyptian population (Bastawisi et al., 2003). In addition, rice constitutes one of the main agricultural exports. The rice area cultivated was 1.424 million feddans 
with an average of $4.00 \mathrm{t} \mathrm{fed}^{-1}$, and a total production of 5.70 million tons over the past five years (RRTC, 2014).

Rice is very sensitive to salinity stress and is currently listed as the most salt sensitive cereal crop with a threshold of $3 \mathrm{dSm}^{-1}$ for most cultivated varieties (USDA, 2013). Rice yield in salt-affected land is significantly reduced with an estimation of $30-50 \%$ yield losses annually (Eynard et al., 2005). Drought is the most significant limiting factor for plant agriculture worldwide, which can cause serious losses of yield and productivity in most crop plants in arid and sub-arid regions. The degree of these effects depends on its impact on the plant physiological, biochemical and the ability of plant to adapt to drought stress (Massonnet et al., 2007). The major environmental factor that constrains the productivity and stability of plants is water stress (Araus et al., 2002). Salinity and drought stresses are among the most serious challenges to crop production in the world today, particularly in developing countries (Zhou et al., 2007). Drought and salinity tolerance can be designated as complex traits due to the interplay of multiple pathways that mediate a plant's capacity to withstand these abiotic stresses (Witcombe et al., 2008).

Molecular markers offer specific advantages in assessment of genetic diversity and in trait specific crop improvement. The use genetic of markers in applied breeding programs can range from facilitating appropriate choice of parents for hybrids. SSR markers are more popular in rice because they are highly informative, mostly monolocus, codominant, easily analyzed and cost effective (Gracia et al., 2004). This investigation aimed to study the GCA and SCA and their interaction with different stress conditions, also to identify some markers associated with drought and salinity tolerance. This study aimed to evaluate some rice genotypes under drought and salt stress using SSR marker linked to those traits.

\section{MATERIALS AND METHODS}

This investigation was carried out in Genetics Department laboratories, Faculty of Agriculture, Kafrelsheikh University, Egypt. Field experiment were carried out at the Rice Research and Training Center (RRTC) facilities at El-Sirw Agriculture Research Station experimental farm, Damietta governorate, Egypt, during 2013 and 2014 rice growing seasons. Eight rice genotypes namely; Sakha102, Sakha104, Sakha105, Sakha106, Giza178, A22, IRAT170, and WAB56-125 were chosen as a parental lines based on the previous studies according to RRTC data 2011. These genotypes have a wide range of variation for salt and drought tolerance due to their different genetic backgrounds. The pedigree, group type and main characters of these varieties are shown in Table (1). Each parent was grown in five 
rows; each row was 5 meter long and contained 25 hills. At flowering the five parents were diallel crossed, i.e., in all possible combinations (excluding reciprocal) to produce $F_{1}$ hybrid seeds following the method proposed by Jodon (1938) and modified by Butany (1961).

Table 1: Name, origin, parentage, type, salinity and drought tolerance of the eight rice varieties

\begin{tabular}{|c|c|c|c|c|c|}
\hline Variety & Origin & Parentage & Type & $\begin{array}{c}\text { Salinity } \\
\text { tolerance }\end{array}$ & $\begin{array}{c}\text { Drought } \\
\text { tolerance }\end{array}$ \\
\hline Sakha102 & Egypt & Giza176/Milyang79 & Japonica & Sensitive & Sensitive \\
\hline Sakha104 & Egypt & $\begin{array}{c}\text { GZ4096-7-1/GZ4120- } \\
\text { 2-5-2 (Giza177) }\end{array}$ & Japonica & Moderate & Moderate \\
\hline Sakha105 & Egypt & $\begin{array}{c}\text { GZ5581-46- } \\
\text { 3/GZ4316-7-1-1 }\end{array}$ & Japonica & Sensitive & Sensitive \\
\hline Sakha106 & Egypt & Giza177/Hexi30 & Japonica & Sensitive & Sensitive \\
\hline Giza178 & Egypt & Giza175/Milyang49 & $\begin{array}{c}\text { Indica/ } \\
\text { Japonica }\end{array}$ & Tolerant & Tolerant \\
\hline A22 & Sri-lank & IR47664 & Indica & Tolerant & Tolerant \\
\hline IRAT170 & $\begin{array}{c}\text { Côte } \\
\text { d'Ivoire }\end{array}$ & IRAT13/Palawan & $\begin{array}{c}\text { Tropical- } \\
\text { Japonica }\end{array}$ & - & Tolerant \\
\hline WAB56-125 & $\begin{array}{c}\text { Côte } \\
\text { d'Ivoire }\end{array}$ & IDSA6 / IAC164 & Indica & - & Tolerant \\
\hline
\end{tabular}

The eight parents and their $28 F_{1}$ hybrid were grown in a randomized complete block design with three replications under three conditions (normal, salinity with ECe $8.88 \mathrm{dsm}^{-1}$ and drought conditions), where drought stress was imposed by using flush irrigation every 10 days without standing water after irrigation. All the data were recorded on the parents and their crosses in three sites. All recommended agricultural practices were applied according to rice research program, Egypt. At ripening, each plant was harvested individually.

\section{Soil analysis:}

The procedure for preparation and measurements of the soil extract was taken according to the method of Black et al. (1965). The methods of Chapman and Parker (1961) of soil chemical analysis were followed. The chemical analysis of experimental sites was listed as shown in Table (2). 
Table 2: Chemical and physical analyses of the experimental sites during 2014

\begin{tabular}{|c|c|c|c|}
\hline \multirow{2}{*}{ Character } & \multicolumn{3}{|c|}{2014} \\
\cline { 2 - 4 } & Normal & Salinity & Drought \\
\hline E.C. & 2.37 & 8.88 & 2.0 \\
\hline P.H. & 8.09 & 8.42 & 8.1 \\
\hline Soluble ions: & \multicolumn{3}{|c|}{$(\mathrm{meq} / \mathrm{L})$} \\
\hline $\mathrm{Ca}^{++}$ & 2.8 & 10.9 & 2 \\
\hline $\mathrm{Mg}^{++}$ & 2.9 & 7.1 & 1 \\
\hline $\mathrm{Na}^{+}$ & 18.02 & 70 & 16.49 \\
\hline $\mathrm{K}^{+}$ & 0.37 & 0.39 & 0.59 \\
\hline $\mathrm{Co}_{3}{ }^{2}$ & 2.07 & 5.08 & 2 \\
\hline $\mathrm{HcO}_{3}{ }^{-}$ & 1.4 & 8 & 1.1 \\
\hline $\mathrm{Cl}^{-}$ & 15.5 & 65 & 12.9 \\
\hline $\mathrm{So}_{4}{ }^{=}$ & 6 & 10 & 4 \\
\hline
\end{tabular}

\section{Studied characters:}

Plants of twenty hills were randomly taken from each parent and $F_{1}$ cross, from each replicate. Data were collected on ten rice traits, viz; days to heading, plant height, total chlorophyll content, proline content, sodium content, potassium content, $\mathrm{Na}^{+} / \mathrm{K}^{+}$ratio, panicles plant ${ }^{-1}$, filled grains panicle ${ }^{-1}$ and grain yield plant $^{-1}$. Where, Proline content was estimated according to Sadasivam and Manickam (1996), furthermore, $\mathrm{Na}^{+}$and $\mathrm{K}^{+}$leaf content were determined by flam photometer according to Richards (1954).

\section{Statistical Analysis:}

Data of the three treatments were subjected to Randomized Complete Block Design (RCBD) according to Snedecor and Cochran (1967). At first, the data were analyzed by using the ordinary analysis of variance to test the significance of differences among the genotypes studied (eight parents and their crosses). If the genotypic mean squares were found to be significant, there was a need to proceed for further analysis; i.e., Griffing (1956) mode 1, method 2. Estimates of combining ability analysis were estimated according to Griffing, 1956 model 1 method 2.

\section{Molecular studies:-}

DNA isolation:-

Genomic DNA was isolated from $0.5 \mathrm{~g}$ of three week old leaves of the used rice genotypes using CTAB method described by (Murray and Thompson, 1980).

\section{SSR markers and PCR amplification:-}

Eight SSR markers i.e. RM 219, RM 201, RM3805, RM 72, RM 223, RM 315, RM8094 and RM 25 were used. The sequences of 
primer pairs are found on the Web database (http://www.gramene.org). PCR amplification reactions were done according to Lodha et al. (2011). Primers names, repeat motifs and chromosome number are shown in Table (3).

\section{SSR data analysis:-}

The amplified bands were scored for each SSR marker based on the presence or absence of bands, generating a binary data matrix of 1 and 0 for each marker system. Effective alleles per locus (Aep) were calculated according to Weir (1989) $($ Aep $=1 /(1-\mathrm{He})$, where Hep, the genetic diversity per locus). Genetic diversity was calculated according to Nei (1973). Polymorphic information content (PIC) values were calculated for each SSR marker by using the formula described by Anderson et al. (1993) as follows:

$(\mathrm{PIC}=1-\Sigma \mathrm{P} i 2$, where $\mathrm{p} i$ is the frequency of the ith allele)

\section{Phylogenetic tree construction:-}

The presence/absence matrix for amplified DNA fragments was analyzed using the PAST program, ver. 1.90 (Hammer et al., 2001). Data matrix was used to calculate genetic similarity based on Jaccard's similarity coefficients, and dendrogram displaying relationships among eight rice genotypes was constructed using the Unweighted Pair Group Method with Arithmetic Mean (UPGMA).

Table 3: Name; chromosome number (CN); SSR motifs and the sequences of the SSR markers used in the current study and related traits

\begin{tabular}{|c|c|c|c|c|c|c|c|}
\hline No. & Markers & $\mathrm{CN}$ & SSR motif & & Sequence & Trait & References \\
\hline \multirow{2}{*}{1} & \multirow{2}{*}{ RM 219} & \multirow{2}{*}{9} & \multirow{2}{*}{$(\mathrm{CT}) 17$} & $\mathrm{~F}$ & CGTCGGATGATGTAAAGCCT & \multirow{2}{*}{ Salinity } & \multirow{2}{*}{$\begin{array}{l}\text { Chatterjee and Reddy } \\
\text { (2014) }\end{array}$} \\
\hline & & & & $\mathrm{R}$ & CATATCGGCATTCGCCTG & & \\
\hline \multirow{2}{*}{2} & \multirow{2}{*}{ RM 201} & \multirow{2}{*}{9} & \multirow{2}{*}{ (CT) 17} & $\mathrm{~F}$ & CGTTTATTACCTACAGTACC & \multirow{2}{*}{ Drought } & \multirow[t]{2}{*}{ Chaitra et al. (2006) } \\
\hline & & & & $\mathrm{R}$ & CTACCTCCTTTCTAGACCGATA & & \\
\hline \multirow{2}{*}{3} & \multirow{2}{*}{ RM3805 } & \multirow{2}{*}{6} & \multirow{2}{*}{$(\mathrm{GA}) 19$} & $\mathrm{~F}$ & AGAGGAAGAAGCCAAGGAGG & \multirow{2}{*}{ Drought } & \multirow[t]{2}{*}{ Ramadan et al. (2015) } \\
\hline & & & & $\mathrm{R}$ & CATCAACGTACCAACCATGG & & \\
\hline \multirow{2}{*}{4} & \multirow{2}{*}{ RM 72} & \multirow{2}{*}{8} & \multirow{2}{*}{ (TAT)5C(ATT) 15} & $\mathrm{~F}$ & CCGGCGATAAAACAATGAG & \multirow{2}{*}{ Drought } & \multirow[t]{2}{*}{ Lin et al. (2007) } \\
\hline & & & & $\mathrm{R}$ & GCATCGGTCCTAACTAAGGG & & \\
\hline \multirow{2}{*}{5} & \multirow{2}{*}{ RM 223} & \multirow{2}{*}{8} & \multirow{2}{*}{ (CT)25 } & $\mathrm{F}$ & GAGTGAGCTTGGGCTGAAAC & \multirow{2}{*}{ Drought } & \multirow[t]{2}{*}{ Kumar et al, (2005) } \\
\hline & & & & $\mathrm{R}$ & GAAGGCAAGTCTTGGCACTG & & \\
\hline \multirow{2}{*}{6} & \multirow{2}{*}{ RM 315} & \multirow{2}{*}{1} & \multirow{2}{*}{ (AT)4(GT) 10} & $\mathrm{~F}$ & GAGGTACTTCCTCCGTTTCAC & \multirow{2}{*}{ Salinity } & \multirow{2}{*}{$\begin{array}{l}\text { Chatterjee and Reddy } \\
\text { (2014) }\end{array}$} \\
\hline & & & & $\mathrm{R}$ & AGTCAGCTCACTGTGCAGTG & & \\
\hline \multirow{2}{*}{7} & & 1 & & $\mathrm{~F}$ & AAGTTTGTACACATCGTATACA & & Mohammadi-Nejad et \\
\hline & RM8094 & 1 & (AI) 31 & $\mathrm{R}$ & CGCGACCAGTACTACTACTA & Salınity & al.(2008) \\
\hline & & & & $\mathrm{F}$ & GGAAAGAATGATCTTTTCATGG & & Matin et al. (2012) \\
\hline 8 & RM 25 & 8 & (GA) 18 & $\mathrm{R}$ & CTACCATCAAAACCAATGTTC & Drought & \\
\hline
\end{tabular}

\section{RESULTS AND DISCUSSION}

\section{Analysis of variance}

Mean squares of genotypes (parents and their crosses) were found to be highly significant for ten characters studied at the three conditions, indicating overall differences among these populations (Table 4). General and specific combining ability variances were found to be highly significant for all characteristics studied under the three conditions. These results would indicate the importance of both 
additive and non-additive genetic variances in determining the performance of these characteristics. GCA/SCA ratio was more than unity for filled grains panicle ${ }^{-1}$ under the three conditions; grain yield plant ${ }^{-1}$ under salinity and drought stress; days to heading under normal condition; $\mathrm{K}^{+}$content and $\mathrm{Na}^{+} / \mathrm{K}^{+}$ratio under drought condition; and panicles plant ${ }^{-1}$ under salinity, suggesting the importance of additive type of gene action in the inheritance of these traits under these environments. On the other hand, GCA/SCA ratio was less than unity for plant height, $\mathrm{Na}^{+}$content, proline content, total chlorophyll under the three conditions; days to heading under salinity and drought conditions; $\mathrm{K}^{+}$content and $\mathrm{Na}^{+} / \mathrm{K}^{+}$ratio under normal and salinity conditions; panicles plant ${ }^{-1}$ under normal and drought; and grain yield plant $^{-1}$ under normal condition suggesting the importance of nonadditive gene action in the inheritance of these traits. It is therefore, could be concluded that selection procedures based on the accumulation of additive effects would be successful in improving these characters. These findings were in agreement with those reported by EL-Mowafi and Abou Shousha (2003), Hammoud et al. (2008), Shehata et al. (2009) and El-Mouhamady et al. (2010).

\section{Mean performance}

Data in Table (5) indicated that, the most desirable mean values among parents were Giza178 for panicles plant ${ }^{-1}$, proline content, sodium content, potassium content, $\mathrm{Na} / \mathrm{k}$ ratio, filled grains panicle $^{-1}$ and grain yield plant ${ }^{-1}$ followed by WAB56-125 and A22 for most traits under three conditions. Moreover, Sakha105 the most desirable mean values among parents for plant height and Sakha102 for days to heading under three conditions. Furthermore, the best crosses for mean values were Sakha102 $\times$ Giza178, Sakha102 $\times$ A22, Sakha102 × Giza178, Sakha102 × Giza178, Giza178 × WAB56-125, A22 $\times$ WAB56-125 and Sakha105× WAB56-125 for chlorophyll content, proline content, sodium content, potassium content, $\mathrm{Na} / \mathrm{K}$ ratio, panicles plant ${ }^{-1}$, filled grains panicls ${ }^{-1}$ and grain yield plant ${ }^{-1}$ under three conditions. While, the most desirable mean values under the three conditions were Sakha105 $\times$ Sakha106, Sakha106 $\times$ IRAT170 for plant height; Sakha102 $\times$ Sakha105, Sakha102 $\times$ Sakha105 for days to heading. Finally these parents and crosses could be used in breeding programs to enhance these characters. These findings were in conformity with that reported by Weerakoon et al. (2008), ElMouhamady (2009), Shehata et al. (2009) and Zayed et al. (2014).

\section{Combining ability:}

General combining ability effects:

Data in Table (6) showed that, the best combiners under normal, salinity and drought conditions were Sakha105 and Sakha106 
for plant height; Sakha102 and Sakha106 for days to heading; Giza178, WAB56-125 and A22 for total chlorophyll content, proline content, sodium content, potassium content, $\mathrm{Na}^{+} / \mathrm{K}^{+}$ratio and panicles plant $^{-1}$; Giza178, WAB56-125 and A22 for filled grains panicle ${ }^{-1}$; Giza178, WAB56-125 and A22 for grain yield plant ${ }^{-1}$. So, this parents could be used as a good combiner for improve this trait under these conditions. Those parents showed better performance under salt and drought stresses that attributed to its high ion selectivity with high ability to proline releasing resulted in high tolerance and improving rice growth as well as yield and yield components. These results were in agreement with those reported by Shehata et al. (2009), ElMouhamady et al. (2010) and Sedeek et al. (2012).

\section{Specific combining ability effects:}

From the results in Table (7), the most desirable hybrids were, Sakha105 X A22, Sakha102 x A22 and Sakha104 x Giza178 for total chlorophyll content, panicles plant ${ }^{-1}$ and grain yield plant $^{-1}$; Giza178 $\mathrm{x}$ WAB56-125 for filled grains panicle ${ }^{-1}$; Sakha102 X Sakha104 for days to heading; Sakha104 X Sakha105 for plant height. These crosses exhibited these results under three studied conditions. Those crosses had the same pattern of their tolerant parents for studied stresses regarding studied traits. Moreover, these crosses could be used in breeding programs to improve this trait under drought and saline soil conditions. These results were reported by Shehata et al. (2009), ElMouhamady et al. (2010) and Sedeek et al. (2012).

Table 4: Mean square estimates of ordinary analysis and combining ability analysis for all traits under normal, salinity and drought conditions

\begin{tabular}{|c|c|c|c|c|c|c|c|c|c|c|c|c|c|c|c|c|}
\hline \multirow{2}{*}{$\begin{array}{l}\text { Sources of } \\
\text { variance }\end{array}$} & \multirow{2}{*}{ df } & \multicolumn{3}{|c|}{ Days to Heading (days) } & \multicolumn{3}{|c|}{ Plant height $(\mathrm{cm})$} & \multicolumn{3}{|c|}{ Panicles plant $^{-1}$} & \multicolumn{3}{|c|}{ Filled grains panicle ${ }^{-1}$} & \multicolumn{3}{|c|}{ Grain yield plant $^{-1}(\mathrm{~g})$} \\
\hline & & $\mathrm{N}$ & $\mathrm{S}$ & $\mathrm{D}$ & $\mathrm{N}$ & $\mathrm{S}$ & $\mathrm{D}$ & $\mathrm{N}$ & $\mathrm{S}$ & $\mathrm{D}$ & $\mathrm{N}$ & $\mathrm{S}$ & $\mathrm{D}$ & $\mathrm{N}$ & $\mathrm{S}$ & $\mathrm{D}$ \\
\hline Replications & 2 & 2.398 & 7.11 & 0.15 & 1.95 & 8.08 & 1.42 & 4.29 & 0.59 & 1.24 & 4.87 & 0.22 & 0.46 & 0.87 & 0.61 & 0.99 \\
\hline Genotypes & 35 & $52.64^{\star \star}$ & $41.71^{\star *}$ & $64.14^{* *}$ & $258.4^{* *}$ & $328.0^{* *}$ & $263.1^{* *}$ & $28.23^{* \star}$ & $59.95^{\star \star}$ & $33.10^{* *}$ & $564.10^{* \star}$ & $612.20^{\star \star}$ & $652.31^{* *}$ & $272.1^{\star \star}$ & $142.5^{\star \star}$ & $140.8^{\star *}$ \\
\hline Parents (P) & 7 & $83.61^{\star *}$ & $48.19^{\star *}$ & $90.26^{* *}$ & $109.5^{\star \star}$ & $145.8^{* *}$ & $143.1^{* *}$ & $20.99^{* *}$ & $82.74^{\star *}$ & $44.05^{\star *}$ & $440.82^{\star \star}$ & $614.54^{\star \star}$ & $895.80^{\star *}$ & $101.9^{\star \star}$ & $156.0^{\star *}$ & $170.0^{* \star}$ \\
\hline Crosses $(C)$ & 27 & $45.26^{* \star}$ & $40.66^{\star *}$ & $58.38^{* *}$ & $275.3^{* *}$ & $371.8^{* *}$ & $251.4^{* *}$ & $31.00^{* *}$ & $52.94^{* *}$ & $31.33^{* *}$ & $616.90^{\star *}$ & $632.97^{* *}$ & $612.90^{\star *}$ & $322.8^{* *}$ & $141.6^{* *}$ & $137.0^{* *}$ \\
\hline P. Vs C. & 1 & $34.98^{\star \star}$ & $24.38^{* *}$ & $36.84^{* *}$ & $843.0^{* *}$ & $421.2^{* *}$ & $1420^{* *}$ & 4.13 & $89.57^{\star \star}$ & $4.12^{\star \star}$ & 1.62 & $35.18^{\star \star}$ & $11.94^{\star *}$ & $94.87^{\star \star}$ & $71.43^{\star *}$ & $36.49^{* *}$ \\
\hline Error & 70 & 1.22 & 3.48 & 1.14 & 3.23 & 2.90 & 4.76 & 1.46 & 0.76 & 0.75 & 1.82 & 1.28 & 2.25 & 0.77 & 0.54 & 0.99 \\
\hline GCA & 7 & $64.88^{\star \star}$ & $44.57^{\star \star}$ & $65.42^{* *}$ & $111.9^{* *}$ & $190.5^{\star *}$ & $146.1^{\star \star}$ & $31.73^{\star \star}$ & $87.36^{\star *}$ & $35.59^{* *}$ & $684.03^{\star \star}$ & $915.88^{* \star}$ & $948.33^{\star *}$ & $282.9^{\star *}$ & $190.4^{\star \star}$ & $167.8^{* *}$ \\
\hline SCA & 28 & $5.72^{\star \star}$ & $6.23^{\star *}$ & $10.37^{* *}$ & $79.67^{* \star}$ & $89.04^{* *}$ & $73.12^{\star \star}$ & $3.83^{\star *}$ & $3.14^{\star *}$ & $4.89^{* *}$ & $64.04^{\star *}$ & $26.12^{* *}$ & $34.72^{\star *}$ & $42.66^{* *}$ & $11.77^{* *}$ & $16.71^{* \star}$ \\
\hline Error & 70 & 0.41 & 1.16 & 0.38 & 1.08 & 0.97 & 1.59 & 0.49 & 0.26 & 0.25 & 0.61 & 0.43 & 0.75 & 0.26 & 0.18 & 0.33 \\
\hline GCA/SCA & & 1.21 & 0.86 & 0.65 & 0.14 & 0.22 & 0.20 & 0.93 & 3.02 & 0.76 & 1.08 & 3.56 & 2.79 & 0.67 & 1.64 & 1.02 \\
\hline
\end{tabular}


Table 4: continue....

\begin{tabular}{|c|c|c|c|c|c|c|c|c|c|c|c|c|c|c|c|c|}
\hline \multirow{2}{*}{$\begin{array}{l}\text { Sources of } \\
\text { variance }\end{array}$} & \multirow[t]{2}{*}{$\mathrm{df}$} & \multicolumn{3}{|c|}{$\begin{array}{l}\text { Total chlorophyll content } \\
\text { (SPAD) }\end{array}$} & \multicolumn{3}{|c|}{$\begin{array}{l}\text { Proline content } \\
(\mu \mathrm{g} / \mathrm{g})\end{array}$} & \multicolumn{3}{|c|}{$\mathrm{Na}^{+} / \mathrm{k}^{+}$ratio } & \multicolumn{3}{|c|}{$\mathrm{Na}^{+}$content $(\%)$} & \multicolumn{3}{|c|}{$\mathrm{K}^{+}$content $(\%)$} \\
\hline & & $\mathrm{N}$ & $S$ & $D$ & $\mathrm{~N}$ & $\mathrm{~s}$ & $\mathrm{D}$ & $\mathrm{N}$ & $\mathrm{S}$ & $D$ & $\mathrm{~N}$ & $\mathrm{~s}$ & $D$ & $\mathrm{~N}$ & $\mathrm{~s}$ & $D$ \\
\hline Replications & 2 & 0.07 & 0.83 & 2.41 & 0.024 & 0.12 & 0.72 & 0.0002 & 0.0001 & 0.0003 & 0.0002 & 0.0007 & 0.0013 & 0.0013 & 0.0001 & 0.0002 \\
\hline Genotypes & 35 & $26.46^{* *}$ & $54.46^{* *}$ & $35.72^{\star \star}$ & $0.756^{\star *}$ & $30.85^{* *}$ & $71.21^{\star *}$ & $0.0100^{* *}$ & $0.2666^{* *}$ & $0.0523^{* \star}$ & $0.0172^{* *}$ & $0.2363^{\star \star}$ & $0.0563^{\star *}$ & $0.1928^{\star *}$ & $0.0560^{\star *}$ & $0.1180^{* *}$ \\
\hline Parents $(\mathrm{P})$ & 7 & $11.13^{\star *}$ & $25.93^{* *}$ & $22.71^{* *}$ & $0.814^{\star *}$ & $36.50^{* *}$ & $59.71^{\star *}$ & $0.0066^{* *}$ & $0.2989^{\star *}$ & $0.0522^{* *}$ & $0.0057^{\star \star}$ & $0.2252^{* \star}$ & $0.0500^{\star *}$ & $0.1177^{\star *}$ & $0.0654^{\star *}$ & $0.1326^{* *}$ \\
\hline Crosses (C) & 27 & $29.92^{\star *}$ & $57.31^{* *}$ & $36.84^{* \star}$ & $0.764^{\star *}$ & $29.43^{\star *}$ & $74.65^{\star *}$ & $0.0103^{* *}$ & $0.2661^{\star *}$ & $0.0541^{* *}$ & $0.0196^{* *}$ & $0.2479^{* *}$ & $0.0589^{* *}$ & $0.2064^{* *}$ & $0.0539^{* *}$ & $0.1182^{* *}$ \\
\hline P. Vs C. & 1 & $40.43^{\star *}$ & $177.4^{* *}$ & $96.51^{* *}$ & 0.134 & $29.73^{\star *}$ & $58.79^{* *}$ & $0.0251^{* *}$ & $0.0541^{* *}$ & $0.0038^{* *}$ & $0.0324^{* *}$ & 0.0010 & $0.0284^{\star *}$ & $0.3511^{* *}$ & $0.0463^{\star *}$ & $0.0109^{* *}$ \\
\hline Error & 70 & 1.71 & 0.70 & 1.49 & 0.055 & 0.33 & 0.46 & 0.0003 & 0.0001 & 0.0007 & 0.0006 & 0.0005 & 0.0013 & 0.0033 & 0.0001 & 0.0019 \\
\hline GCA & 7 & $10.63^{* *}$ & $52.96^{* *}$ & $29.45^{\star *}$ & $0.662^{* *}$ & $30.40^{* *}$ & $65.51^{\star *}$ & $0.0100^{* *}$ & $0.3105^{\star *}$ & $0.0633^{* *}$ & $0.0116^{* *}$ & $0.2321^{\star *}$ & $0.0551^{\star *}$ & $0.1964^{* *}$ & $0.0637^{* \star}$ & $0.1460^{\star *}$ \\
\hline SCA & 28 & $8.37^{\star \star}$ & $9.45^{\star *}$ & $7.52^{\star *}$ & $0.149^{* *}$ & $5.25^{\star \star}$ & $13.29^{* *}$ & $0.0017^{\star *}$ & $0.0335^{\star \star}$ & $0.0060^{* *}$ & $0.0043^{* *}$ & $0.0404^{* *}$ & $0.0097^{\star *}$ & $0.0312^{\star *}$ & $0.0074^{\star *}$ & $0.0127^{\star *}$ \\
\hline Error & 70 & $0.57^{\star \star}$ & 0.23 & 0.50 & 0.018 & 0.11 & 0.15 & 0.0001 & 0.0001 & 0.0002 & 0.0002 & 0.0002 & 0.0004 & 0.0011 & 0.0001 & 0.0006 \\
\hline GCA/SCA & & 0.13 & 0.57 & 0.41 & 0.49 & 0.59 & 0.50 & 0.64 & 0.93 & 1.10 & 0.28 & 0.58 & 0.59 & 0.65 & 0.86 & 1.21 \\
\hline
\end{tabular}

* and ${ }^{* *}$ : Significant at 0.05 and 0.01 levels of probability, respectively, $\mathrm{N}=$ Normal environment,

$\mathrm{S}=$ salinity and $\mathrm{D}=$ Drought environment.

Table 5: Mean performance of ten traits in the studied rice genotypes under normal, salinity and drought conditions

\begin{tabular}{|c|c|c|c|c|c|c|c|c|c|c|c|c|c|c|c|}
\hline \multirow{2}{*}{ Genotypes } & \multicolumn{3}{|c|}{ Days to Heading (days) } & \multicolumn{3}{|c|}{ Plant height $(\mathrm{cm})$} & \multicolumn{3}{|c|}{ Panicles plant ${ }^{-1}$} & \multicolumn{3}{|c|}{ Filled grains panicle ${ }^{-1}$} & \multicolumn{3}{|c|}{ Grain yield plant $^{-1}(\mathrm{~g})$} \\
\hline & $\mathrm{N}$ & $\mathrm{S}$ & $\mathrm{D}$ & $\mathrm{N}$ & $\mathrm{S}$ & $\mathrm{D}$ & $\mathrm{N}$ & $\mathrm{S}$ & $\mathrm{D}$ & $\mathrm{N}$ & $\mathrm{S}$ & $\mathrm{D}$ & $\mathrm{N}$ & $\mathrm{S}$ & $\mathrm{D}$ \\
\hline Sakha102 & 93.00 & 94.33 & 92.00 & 101.0 & 69.00 & 80.00 & 17.33 & 6.00 & 9.67 & 99.85 & 56.17 & 65.33 & 34.67 & 6.34 & 12.33 \\
\hline Sakha104 & 107.0 & 105.3 & 103.3 & 99.00 & 76.67 & 83.00 & 19.33 & 10.33 & 13.67 & 101.8 & 60.19 & 70.22 & 40.77 & 12.46 & 17.11 \\
\hline Sakha105 & 96.67 & 97.33 & 93.00 & 92.00 & 67.33 & 70.00 & 18.33 & 4.00 & 8.53 & 98.14 & 55.15 & 60.33 & 36.62 & 5.67 & 11.21 \\
\hline Sakha106 & 95.33 & 96.67 & 91.00 & 95.67 & 71.33 & 74.00 & 18.67 & 6.667 & 12.23 & 104.8 & 53.35 & 62.33 & 37.72 & 7.90 & 14.50 \\
\hline Giza178 & 104.3 & 104.7 & 103.0 & 94.33 & 86.00 & 90.00 & 23.33 & 17.33 & 19.00 & 128.5 & 85.98 & 101.3 & 49.32 & 23.33 & 30.33 \\
\hline A22 & 106.0 & 103.3 & 104.0 & 98.33 & 83.67 & 87.33 & 20.00 & 15.33 & 18.00 & 120.3 & 84.43 & 95.67 & 47.29 & 21.43 & 26.90 \\
\hline IRAT170 & 103.7 & 101.3 & 100.7 & 112.0 & 75.33 & 84.33 & 15.33 & 4.333 & 13.00 & 115.2 & 56.59 & 88.25 & 34.00 & 8.37 & 20.24 \\
\hline WAB56-125 & 102.0 & 101.0 & 100.3 & 99.33 & 82.00 & 86.80 & 22.67 & 14.00 & 17.00 & 125.5 & 80.15 & 97.67 & 44.33 & 19.35 & 28.23 \\
\hline Sakha102 X Sakha104 & 98.33 & 98.00 & 93.67 & 97.33 & 87.00 & 88.33 & 17.00 & 8.67 & 12.33 & 98.83 & 65.15 & 67.96 & 34.60 & 9.17 & 16.17 \\
\hline Sakha102 X Sakha105 & 97.67 & 96.67 & 95.00 & 95.33 & 66.00 & 82.33 & 14.67 & 5.67 & 7.33 & 89.33 & 48.67 & 60.43 & 25.67 & 5.08 & 10.78 \\
\hline Sakha102 X Sakha106 & 93.00 & 94.00 & 92.67 & 97.00 & 66.00 & 80.00 & 16.00 & 6.00 & 10.67 & 95.26 & 52.33 & 63.25 & 30.80 & 5.17 & 12.55 \\
\hline Sakha102 X Giza178 & 100.7 & 98.00 & 96.33 & 114.3 & 86.00 & 100.7 & 24.33 & 15.33 & 18.67 & 114.7 & 73.33 & 88.67 & 55.33 & 20.95 & 24.91 \\
\hline Sakha102 X A22 & 104.3 & 102.3 & 99.33 & 117.7 & 97.67 & 100.0 & 23.67 & 14.33 & 18.33 & 116.0 & 77.51 & 84.26 & 51.93 & 21.46 & 28.16 \\
\hline Sakha102 X IRAT170 & 102.7 & 102.3 & 100.7 & 116.0 & 62.33 & 97.00 & 18.33 & 5.00 & 13.00 & 100.5 & 56.29 & 78.25 & 39.17 & 4.96 & 21.14 \\
\hline Sakha102 X WAB56-125 & 99.67 & 99.00 & 96.00 & 114.0 & 88.33 & 99.00 & 19.67 & 13.33 & 14.67 & 124.0 & 77.26 & 84.19 & 43.33 & 20.45 & 26.21 \\
\hline Sakha104 X Sakha105 & 101.0 & 102.3 & 99.67 & 93.00 & 68.67 & 71.00 & 17.67 & 8.33 & 11.33 & 97.62 & 49.99 & 53.48 & 26.12 & 6.82 & 9.97 \\
\hline Sakha104 X Sakha106 & 103.0 & 104.3 & 104.0 & 94.33 & 68.67 & 72.33 & 16.00 & 8.67 & 9.33 & 87.85 & 48.55 & 53.67 & 25.71 & 6.41 & 8.00 \\
\hline Sakha104 X Giza178 & 106.7 & 104.3 & 105.7 & 117.3 & 90.00 & 93.33 & 24.67 & 17.67 & 18.33 & 115.8 & 82.91 & 85.25 & 51.73 & 26.47 & 28.63 \\
\hline Sakha104 X A22 & 110.3 & 110.0 & 109.3 & 107.3 & 95.33 & 97.67 & 21.67 & 18.33 & 16.00 & 130.5 & 81.19 & 88.67 & 47.33 & 21.69 & 24.17 \\
\hline Sakha104 X IRAT170 & 108.0 & 107.0 & 106.0 & 107.3 & 85.67 & 91.67 & 16.67 & 9.67 & 11.33 & 116.6 & 60.23 & 83.29 & 38.67 & 11.24 & 20.89 \\
\hline Sakha104 X WAB56-125 & 109.0 & 108.0 & 108.3 & 98.67 & 90.33 & 93.00 & 19.67 & 15.67 & 15.67 & 127.6 & 78.67 & 88.30 & 45.22 & 22.63 & 27.21 \\
\hline Sakha105 X Sakha106 & 98.67 & 100.0 & 97.33 & 85.33 & 68.67 & 71.00 & 18.00 & 5.67 & 10.00 & 87.64 & 43.35 & 55.29 & 27.95 & 6.63 & 12.67 \\
\hline Sakha105 X Giza178 & 100.0 & 98.33 & 98.00 & 106.0 & 80.67 & 93.67 & 21.33 & 12.67 & 15.67 & 111.2 & 63.56 & 70.67 & 44.24 & 17.92 & 21.33 \\
\hline Sakha105 X A22 & 102.0 & 101.3 & 100.7 & 120.3 & 96.00 & 102.3 & 23.67 & 14.00 & 18.33 & 114.5 & 65.74 & 77.29 & 58.33 & 18.56 & 30.65 \\
\hline Sakha105 X IRAT170 & 107.0 & 102.7 & 100.7 & 101.0 & 79.00 & 81.67 & 14.67 & 5.67 & 10.00 & 108.7 & 55.73 & 78.67 & 30.90 & 6.43 & 15.77 \\
\hline Sakha105 X WAB56-125 & 104.7 & 105.3 & 103.0 & 113.0 & 88.00 & 96.33 & 23.33 & 11.33 & 15.00 & 112.0 & 67.26 & 74.67 & 54.92 & 13.14 & 22.13 \\
\hline Sakha106 X Giza178 & 97.67 & 98.67 & 98.00 & 105.3 & 78.33 & 96.67 & 20.33 & 13.67 & 16.67 & 115.3 & 68.67 & 72.22 & 40.59 & 16.20 & 17.75 \\
\hline Sakha106 X A22 & 100.3 & 103.0 & 98.67 & 116.3 & 98.67 & 104.3 & 21.67 & 15.67 & 17.33 & 115.3 & 68.67 & 79.33 & 51.67 & 21.10 & 25.36 \\
\hline Sakha106 X IRAT170 & 99.00 & 98.67 & 96.67 & 99.33 & 60.00 & 87.67 & 15.67 & 6.67 & 11.33 & 106.7 & 53.15 & 81.67 & 40.48 & 7.13 & 16.85 \\
\hline Sakha106 X WAB56-125 & 103.3 & 102.7 & 100.7 & 109.0 & 80.33 & 98.00 & 18.00 & 12.33 & 16.33 & 111.3 & 64.48 & 76.67 & 38.40 & 14.60 & 20.68 \\
\hline Giza178 X A22 & 103.7 & 98.00 & 96.00 & 97.67 & 81.00 & 85.00 & 22.33 & 17.00 & 13.33 & 148.5 & 94.26 & 95.22 & 56.00 & 18.81 & 21.74 \\
\hline Giza178 X IRAT170 & 100.7 & 98.33 & 93.33 & 114.7 & 86.00 & 95.33 & 19.67 & 15.33 & 17.00 & 111.3 & 73.67 & 89.15 & 41.97 & 20.31 & 32.06 \\
\hline Giza178 X WAB56-125 & 102.3 & 102.7 & 97.00 & 98.00 & 76.33 & 89.00 & 23.00 & 17.00 & 18.33 & 135.9 & 99.94 & 110.7 & 54.11 & 25.24 & 32.90 \\
\hline A22 X IRAT170 & 105.0 & 105.3 & 104.7 & 119.0 & 86.00 & 89.67 & 21.00 & 13.33 & 13.67 & 109.3 & 64.67 & 85.67 & 49.33 & 15.65 & 21.76 \\
\hline A22 X WAB56-125 & 105.7 & 104.0 & 101.7 & 96.00 & 71.00 & 89.33 & 25.33 & 15.67 & 15.00 & 128.5 & 93.97 & 110.1 & 57.60 & 20.12 & 26.05 \\
\hline IRAT170 X WAB56-125 & 102.0 & 100.7 & 102.0 & 108.3 & 90.67 & 92.00 & 17.67 & 11.67 & 17.00 & 106.8 & 71.22 & 84.67 & 37.52 & 17.37 & 25.63 \\
\hline LSD 0.05 & 1.80 & 3.05 & 1.74 & 2.93 & 2.78 & 3.56 & 1.97 & 1.43 & 1.42 & 2.20 & 1.84 & 2.45 & 1.44 & 1.20 & 1.62 \\
\hline LSD 0.01 & 2.40 & 4.05 & 2.33 & 3.90 & 3.70 & 4.74 & 2.62 & 1.90 & 1.89 & 2.93 & 2.45 & 3.26 & 1.91 & 1.59 & 2.16 \\
\hline
\end{tabular}

$\mathrm{N}=$ normal $\mathrm{s}=$ salinity $\mathrm{d}=\mathrm{drought}$ 
Table 5: Continue......

\begin{tabular}{|c|c|c|c|c|c|c|c|c|c|c|c|c|c|c|c|}
\hline \multirow{2}{*}{$\begin{array}{ll} & \text { Traits } \\
\text { Genotypes } & \\
\end{array}$} & \multicolumn{3}{|c|}{$\begin{array}{l}\text { Total chlorophyll content } \\
\text { (SPAD) }\end{array}$} & \multicolumn{3}{|c|}{ Proline content $(\mu \mathrm{g} / \mathrm{g})$} & \multicolumn{3}{|c|}{$\mathrm{Na}^{+} / \mathrm{k}^{+}$ratio } & \multicolumn{3}{|c|}{$\mathrm{Na}^{+}$content $(\%)$} & \multicolumn{3}{|c|}{$\mathrm{K}^{+}$content $(\%)$} \\
\hline & $\mathrm{N}$ & $S$ & $\mathrm{D}$ & $\mathrm{N}$ & $\mathrm{s}$ & $\mathrm{D}$ & $\mathrm{N}$ & $\mathrm{s}$ & $\mathrm{D}$ & $\mathrm{N}$ & $\mathrm{S}$ & $\mathrm{D}$ & $\mathrm{N}$ & $\mathrm{s}$ & $\mathrm{D}$ \\
\hline Sakha102 & 45.63 & 33.60 & 39.00 & 1.81 & 11.17 & 14.70 & 0.304 & 1.641 & 0.681 & 0.591 & 2.024 & 1.093 & 1.944 & 1.234 & 1.605 \\
\hline Sakha104 & 48.20 & 38.63 & 40.60 & 2.40 & 14.67 & 19.43 & 0.292 & 1.252 & 0.551 & 0.526 & 1.700 & 0.904 & 1.802 & 1.359 & 1.641 \\
\hline Sakha105 & 44.17 & 34.43 & 36.33 & 1.40 & 8.90 & 13.47 & 0.352 & 1.867 & 0.698 & 0.574 & 2.381 & 1.043 & 1.633 & 1.276 & 1.495 \\
\hline Sakha106 & 46.67 & 35.40 & 37.23 & 2.07 & 11.33 & 17.15 & 0.299 & 1.457 & 0.655 & 0.533 & 1.878 & 0.991 & 1.782 & 1.290 & 1.514 \\
\hline Giza178 & 49.27 & 41.83 & 43.33 & 2.95 & 19.10 & 24.70 & 0.208 & 0.955 & 0.357 & 0.465 & 1.556 & 0.730 & 2.238 & 1.630 & 2.046 \\
\hline A22 & 49.53 & 40.73 & 42.33 & 2.30 & 17.87 & 20.03 & 0.240 & 1.063 & 0.398 & 0.505 & 1.595 & 0.758 & 2.104 & 1.501 & 1.907 \\
\hline IRAT170 & 48.90 & 36.40 & 41.63 & 1.87 & 13.60 & 18.40 & 0.297 & 1.384 & 0.568 & 0.546 & 1.816 & 0.952 & 1.842 & 1.312 & 1.675 \\
\hline WAB56-125 & 48.53 & 37.53 & 43.67 & 2.81 & 15.57 & 26.30 & 0.234 & 1.055 & 0.443 & 0.481 & 1.645 & 0.865 & 2.054 & 1.559 & 1.952 \\
\hline $\begin{array}{ll}\text { Sakha102 } & \text { X } \\
\text { Sakha104 } & \end{array}$ & 47.50 & 35.77 & 37.67 & 2.20 & 10.50 & 15.50 & 0.294 & 1.228 & 0.671 & 0.551 & 1.801 & 1.007 & 1.873 & 1.466 & 1.501 \\
\hline $\begin{array}{ll}\text { Sakha102 } & \text { X } \\
\text { Sakha105 } & \\
\end{array}$ & 41.67 & 32.67 & 37.67 & 1.77 & 9.73 & 10.27 & 0.330 & 1.463 & 0.778 & 0.595 & 2.072 & 1.175 & 1.808 & 1.416 & 1.511 \\
\hline $\begin{array}{l}\text { Sakha102 } \\
\text { Sakha106 }\end{array}$ & 40.97 & 35.50 & 35.20 & 2.57 & 11.73 & 13.43 & 0.262 & 1.299 & 0.708 & 0.522 & 1.824 & 1.075 & 1.994 & 1.405 & 1.522 \\
\hline $\begin{array}{ll}\text { Sakha102 } & X \\
\text { Giza178 } & \end{array}$ & 51.43 & 46.00 & 46.33 & 2.64 & 16.13 & 17.53 & 0.153 & 1.123 & 0.366 & 0.405 & 1.737 & 0.697 & 2.647 & 1.547 & 1.905 \\
\hline Sakha102 X A22 & 48.13 & 45.00 & 45.63 & 2.84 & 12.83 & 22.63 & 0.192 & 1.167 & 0.487 & 0.461 & 1.754 & 0.855 & 2.402 & 1.503 & 1.755 \\
\hline $\begin{array}{l}\text { Sakha102 } \\
\text { IRAT170 }\end{array}$ & 45.60 & 39.50 & 41.33 & 1.67 & 10.27 & 19.40 & 0.258 & 1.627 & 0.499 & 0.522 & 2.201 & 0.925 & 2.026 & 1.352 & 1.853 \\
\hline $\begin{array}{ll}\text { Sakha102 } & \text { X } \\
\text { WAB56-125 } & \\
\end{array}$ & 46.73 & 42.33 & 43.67 & 2.10 & 16.73 & 19.70 & 0.178 & 0.971 & 0.404 & 0.427 & 1.419 & 0.757 & 2.402 & 1.461 & 1.874 \\
\hline $\begin{array}{ll}\text { Sakha104 } & \text { X } \\
\text { Sakha105 } & \end{array}$ & 45.98 & 38.57 & 39.33 & 1.77 & 14.77 & 17.83 & 0.335 & 1.393 & 0.709 & 0.629 & 1.820 & 1.132 & 1.876 & 1.306 & 1.597 \\
\hline $\begin{array}{l}\text { Sakha104 } \\
\text { Sakha106 }\end{array}$ & 40.37 & 34.00 & 38.67 & 1.44 & 13.83 & 15.13 & 0.317 & 1.549 & 0.707 & 0.587 & 2.173 & 1.123 & 1.849 & 1.403 & 1.589 \\
\hline $\begin{array}{ll}\text { Sakha104 } & \mathrm{X} \\
\text { Giza178 } & \end{array}$ & 42.87 & 39.70 & 38.30 & 2.87 & 16.67 & 29.17 & 0.177 & 0.752 & 0.389 & 0.365 & 1.191 & 0.743 & 2.065 & 1.583 & 1.907 \\
\hline Sakha104 X A22 & 39.13 & 37.90 & 38.90 & 2.73 & 15.57 & 22.43 & 0.190 & 1.090 & 0.520 & 0.386 & 1.644 & 0.958 & 2.026 & 1.508 & 1.842 \\
\hline $\begin{array}{ll}\text { Sakha104 } & X \\
\text { IRAT170 } & \end{array}$ & 45.93 & 35.63 & 42.60 & 2.40 & 14.77 & 24.37 & 0.289 & 1.711 & 0.650 & 0.544 & 2.055 & 1.040 & 1.878 & 1.201 & 1.618 \\
\hline $\begin{array}{ll}\text { Sakha104 } & X \\
\text { WAB56-125 } & \\
\end{array}$ & 41.40 & 37.20 & 40.63 & 2.97 & 17.57 & 21.27 & 0.149 & 1.313 & 0.478 & 0.288 & 1.865 & 0.861 & 1.931 & 1.420 & 1.803 \\
\hline $\begin{array}{ll}\text { Sakha105 } & \text { X } \\
\text { Sakha106 } & \\
\end{array}$ & 45.20 & 36.63 & 38.33 & 1.54 & 11.37 & 12.80 & 0.321 & 1.698 & 0.887 & 0.499 & 2.069 & 1.260 & 1.610 & 1.218 & 1.421 \\
\hline $\begin{array}{ll}\text { Sakha105 } & \mathrm{X} \\
\text { Giza178 } & \\
\end{array}$ & 49.00 & 45.43 & 46.20 & 3.10 & 19.60 & 25.50 & 0.168 & 1.159 & 0.466 & 0.377 & 1.684 & 0.822 & 2.241 & 1.453 & 1.766 \\
\hline Sakha105 X A22 & 49.97 & 44.43 & 45.77 & 2.40 & 18.53 & 23.33 & 0.194 & 1.106 & 0.501 & 0.454 & 1.568 & 0.977 & 2.336 & 1.418 & 1.950 \\
\hline $\begin{array}{ll}\text { Sakha105 } & \text { X } \\
\text { IRAT170 } & \\
\end{array}$ & 45.90 & 33.27 & 44.07 & 1.67 & 15.13 & 18.63 & 0.299 & 1.878 & 0.707 & 0.537 & 2.481 & 1.086 & 1.795 & 1.321 & 1.541 \\
\hline $\begin{array}{ll}\text { Sakha105 } & \text { X } \\
\text { WAB56-125 } & \\
\end{array}$ & 47.73 & 42.80 & 45.47 & 1.71 & 15.67 & 20.80 & 0.233 & 1.170 & 0.598 & 0.428 & 1.820 & 1.004 & 1.836 & 1.555 & 1.680 \\
\hline $\begin{array}{ll}\text { Sakha106 } & \text { X } \\
\text { Giza178 } & \end{array}$ & 50.93 & 44.63 & 46.40 & 2.97 & 21.77 & 27.97 & 0.256 & 1.188 & 0.411 & 0.536 & 1.721 & 0.765 & 2.093 & 1.448 & 1.861 \\
\hline Sakha106 X A22 & 48.43 & 43.13 & 46.83 & 2.22 & 17.90 & 26.70 & 0.274 & 1.061 & 0.477 & 0.524 & 1.725 & 0.804 & 1.915 & 1.627 & 1.684 \\
\hline $\begin{array}{l}\text { Sakha106 } \\
\text { IRAT170 }\end{array}$ & 46.70 & 36.73 & 43.10 & 1.52 & 14.57 & 21.63 & 0.308 & 1.914 & 0.666 & 0.620 & 2.381 & 1.097 & 2.011 & 1.244 & 1.647 \\
\hline $\begin{array}{ll}\text { Sakha106 } & X \\
\text { WAB56-125 } & \end{array}$ & 48.10 & 42.00 & 46.03 & 2.74 & 16.70 & 23.70 & 0.250 & 1.283 & 0.549 & 0.466 & 1.823 & 0.984 & 1.863 & 1.421 & 1.791 \\
\hline Giza178 X A22 & 48.10 & 47.47 & 47.80 & 2.07 & 17.57 & 18.90 & 0.182 & 0.989 & 0.410 & 0.427 & 1.580 & 0.880 & 2.349 & 1.598 & 2.149 \\
\hline $\begin{array}{ll}\text { Giza178 } & \mathrm{X} \\
\text { IRAT170 } & \\
\end{array}$ & 46.03 & 44.33 & 43.87 & 2.44 & 15.20 & 25.73 & 0.286 & 1.098 & 0.611 & 0.513 & 1.539 & 0.980 & 1.796 & 1.402 & 1.604 \\
\hline $\begin{array}{ll}\text { Giza178 } & X \\
\text { WAB56-125 } & \end{array}$ & 46.27 & 44.30 & 44.30 & 2.57 & 16.47 & 23.53 & 0.203 & 0.856 & 0.447 & 0.489 & 1.564 & 0.882 & 2.415 & 1.827 & 1.975 \\
\hline A22 X IRAT170 & 45.33 & 42.87 & 44.40 & 1.94 & 13.37 & 18.30 & 0.257 & 1.079 & 0.512 & 0.533 & 1.627 & 1.017 & 2.074 & 1.508 & 1.987 \\
\hline A22 X WAB56-125 & 48.43 & 45.30 & 45.33 & 2.53 & 21.30 & 30.80 & 0.173 & 1.089 & 0.418 & 0.447 & 1.666 & 0.917 & 2.581 & 1.530 & 2.193 \\
\hline $\begin{array}{l}\text { IRAT170 } \\
\text { WAB56-125 }\end{array}$ & 48.10 & 38.20 & 44.30 & 2.64 & 11.80 & 22.33 & 0.232 & 1.588 & 0.602 & 0.475 & 2.075 & 0.947 & 2.047 & 1.306 & 1.574 \\
\hline LSD 0.05 & 2.13 & 1.37 & 1.99 & 0.38 & 0.94 & 1.10 & 0.030 & 0.019 & 0.044 & 0.040 & 0.035 & 0.059 & 0.094 & 0.016 & 0.072 \\
\hline LSD 0.01 & 2.84 & 1.82 & 2.65 & 0.51 & 1.26 & 1.47 & 0.040 & 0.026 & 0.059 & 0.053 & 0.046 & 0.078 & 0.126 & 0.021 & 0.096 \\
\hline
\end{tabular}

$\mathrm{N}=$ normal $\mathrm{s}=$ salinity $\mathrm{d}=$ drought 
Table 6: Estimates of general combining ability (GCA) effects for all traits for eight parents under normal, salinity and drought conditions

\begin{tabular}{|c|c|c|c|c|c|c|c|c|c|c|c|c|c|c|c|}
\hline \multirow[t]{2}{*}{$\begin{array}{l}\text { Traits } \\
\text { Parents }\end{array}$} & \multicolumn{3}{|c|}{ Days to Heading (days) } & \multicolumn{3}{|c|}{ Plant height (cm) } & \multicolumn{3}{|c|}{ Panicles plant ${ }^{-1}$} & \multicolumn{3}{|c|}{ Filled grains panicle $^{-1}$} & \multicolumn{3}{|c|}{ Grain yield plant ${ }^{-1}(\mathrm{~g})$} \\
\hline & $\mathrm{N}$ & $\mathrm{S}$ & D & $\mathrm{N}$ & $\mathrm{S}$ & $\mathrm{D}$ & $\mathrm{N}$ & $\mathrm{S}$ & $\mathrm{D}$ & $\mathrm{N}$ & $\mathrm{S}$ & $\mathrm{D}$ & $\mathrm{N}$ & $\mathrm{S}$ & $\mathrm{D}$ \\
\hline Sakha102 & $-3.63^{\star *}$ & $-3.35^{\star \star}$ & $-3.79^{* *}$ & $1.60^{* *}$ & $-2.96^{* *}$ & $0.89^{* *}$ & $-0.93^{* *}$ & $-2.28^{* *}$ & $-1.39^{* *}$ & $-6.96^{\star *}$ & $-4.52^{\star \star}$ & $-5.80^{\star *}$ & $-3.09^{* *}$ & $-3.17^{\star *}$ & $-2.62^{\star *}$ \\
\hline Sakha104 & $3.18^{* *}$ & $3.22^{* *}$ & $3.77^{* *}$ & $-2.43^{* *}$ & $1.80^{* \star}$ & $-2.51^{* *}$ & $-0.57^{* *}$ & $0.46^{* *}$ & $-0.66^{* *}$ & $-2.94^{\star \star}$ & $-2.10^{* *}$ & $-5.46^{* *}$ & $-3.02^{* *}$ & $-0.23^{* *}$ & $-2.15^{\star *}$ \\
\hline Sakha105 & $-1.43^{* *}$ & $-1.12^{\star \star}$ & $-1.53^{* *}$ & $-3.97^{\star \star}$ & $-3.93^{\star *}$ & $-6.01^{\star *}$ & $-0.77^{\star *}$ & $-3.18^{* *}$ & $-2.35^{\star \star}$ & $-9.07^{\star *}$ & $-10.35^{\star *}$ & $-12.45^{\star *}$ & $-3.97^{* *}$ & $-4.57^{\star *}$ & $-4.50^{* *}$ \\
\hline Sakha106 & $-3.29^{\star *}$ & $-1.78^{\star \star}$ & $-2.56^{\star *}$ & $-3.97^{\star \star}$ & $-5.77^{\star \star}$ & $-4.05^{\star \star}$ & $-1.47^{\star *}$ & $-2.11^{\star \star}$ & $-1.21^{* *}$ & $-7.90^{\star *}$ & $-10.22^{\star \star}$ & $-10.89^{* *}$ & $-5.00^{\star *}$ & $-3.86^{\star \star}$ & $-4.79^{* *}$ \\
\hline Giza178 & 0.18 & $-0.48^{* \star}$ & $-0.52^{* *}$ & $0.43^{*}$ & $2.93^{* *}$ & $3.52^{\star \star}$ & $2.47^{* *}$ & $4.03^{* *}$ & $2.77^{\star *}$ & $10.17^{\star *}$ & $12.02^{* *}$ & $9.88^{* *}$ & $6.15^{\star \star}$ & $6.09^{* *}$ & $4.93^{* *}$ \\
\hline A22 & $2.48^{\star \star}$ & $1.82^{\star \star}$ & $2.27^{\star \star}$ & $3.33^{* *}$ & $7.20^{\star \star}$ & $4.46^{\star \star}$ & $2.17^{\star \star}$ & $3.59^{\star *}$ & $1.97^{\star \star}$ & $9.54^{\star \star}$ & $10.68^{\star \star}$ & $9.62^{* \star}$ & $8.57^{\star \star}$ & $4.86^{\star \star}$ & $4.09^{* \star}$ \\
\hline IRAT170 & $1.31^{* \star}$ & $0.52^{* \star}$ & $0.97^{* \star}$ & $5.20^{* *}$ & $-2.07^{\star \star}$ & $0.52^{*}$ & $-2.33^{* *}$ & $-2.71^{\star \star}$ & $-0.89^{* *}$ & $-1.77^{\star *}$ & $-6.00^{* \star}$ & $4.22^{* \star}$ & $-3.51^{* *}$ & $-3.18^{* *}$ & $0.38^{* *}$ \\
\hline WAB56-125 & $1.21^{\star \star}$ & $1.18^{\star \star}$ & $1.38^{\star \star}$ & -0.20 & $2.80^{\star \star}$ & $3.18^{\star \star}$ & $1.43^{\star \star}$ & $2.19^{\star \star}$ & $1.77^{\star \star}$ & $8.92^{\star \star}$ & $10.50^{* *}$ & $10.89^{* \star}$ & $3.87^{\star *}$ & $4.06^{\star \star}$ & $4.65^{* \star}$ \\
\hline S.E. (gi) & 0.19 & 0.32 & 0.18 & 0.31 & 0.29 & 0.37 & 0.21 & 0.15 & 0.15 & 0.23 & 0.19 & 0.26 & 0.15 & 0.13 & 0.17 \\
\hline S.E.(gi-gi) & 0.28 & 0.48 & 0.28 & 0.46 & 0.44 & 0.56 & 0.31 & 0.23 & 0.22 & 0.35 & 0.29 & 0.39 & 0.23 & 0.19 & 0.26 \\
\hline
\end{tabular}

Table 6: continue....

\begin{tabular}{|c|c|c|c|c|c|c|c|c|c|c|c|c|c|c|c|}
\hline \multirow{2}{*}{$\begin{array}{r}\text { Traits } \\
\text { Parents }\end{array}$} & \multicolumn{3}{|c|}{$\begin{array}{l}\text { Total chlorophyll content } \\
\text { (SPAD) }\end{array}$} & \multicolumn{3}{|c|}{ Proline content $(\mu \mathrm{g} / \mathrm{g})$} & \multicolumn{3}{|c|}{$\mathrm{Na}^{+} / \mathrm{k}^{+}$ratio } & \multicolumn{3}{|c|}{$\mathrm{Na}^{+}$content (\%) } & \multicolumn{3}{|c|}{$\mathrm{K}^{+}$content (\%) } \\
\hline & $\mathrm{N}$ & $\mathrm{S}$ & $\mathrm{D}$ & $\mathrm{N}$ & $S$ & D & $\mathrm{N}$ & $S$ & $\mathrm{D}$ & $\mathrm{N}$ & $S$ & D & $\mathrm{N}$ & $S$ & D \\
\hline Sakha102 & $0.49^{* *}$ & $\overline{-} 1.35^{\star \star}$ & $\overline{-}$ & $0 . \overline{1}$ & $2.48^{\star *}$ & $3.80^{* *}$ & $0.003^{*}$ & $0.053^{* *}$ & $0.028^{* *}$ & $0.021^{* *}$ & $0.049^{* \star}$ & $0.015^{* *}$ & $0.076^{* *}$ & $0.028^{\star *}$ & $0.060^{\star *}$ \\
\hline Sakha104 & $\frac{-}{1.86^{* *}}$ & $\begin{array}{c}- \\
2.14^{* *}\end{array}$ & $2.33^{* *}$ & $0.08^{* *}$ & $0.21^{\star *}$ & $-0.13^{\star}$ & $0.009^{* *}$ & $0.009^{* *}$ & $0.023^{* *}$ & $0.006^{\star \star}$ & $0.042^{* *}$ & $0.014^{* *}$ & $0.118^{* *}$ & $0.030^{* *}$ & $0.060^{* *}$ \\
\hline Sakha105 & $0.44^{* *}$ & $1.48^{* *}$ & $\overline{-}$ & $0 . \overline{3} 6^{* *}$ & $\overline{-}$ & $2.98^{\star \star}$ & $0.034^{\star *}$ & $0.197^{\star \star}$ & $0.105^{\star *}$ & $0.021^{\star *}$ & $0.191^{\star *}$ & $0.102^{* *}$ & $0 . \overline{152^{* *}}$ & $0.066^{* *}$ & $0.128^{* *}$ \\
\hline Sakha106 & $\begin{array}{c}- \\
0.42^{* *}\end{array}$ & $\begin{array}{c}- \\
1.40^{* *}\end{array}$ & $\begin{array}{c}- \\
1.15^{* *}\end{array}$ & $\begin{array}{c}- \\
0.13^{* *}\end{array}$ & $\begin{array}{c}- \\
0.45^{\star *}\end{array}$ & $\begin{array}{c}- \\
1.02^{* *}\end{array}$ & $0.034^{\star \star}$ & $0.128^{* *}$ & $0.072^{\star *}$ & $0.036^{* *}$ & $0.110^{\star \star}$ & $0.056^{* *}$ & $\begin{array}{c}- \\
0.139^{* *}\end{array}$ & $\begin{array}{c}- \\
0.056^{* *}\end{array}$ & $\begin{array}{c}- \\
0.119^{* *}\end{array}$ \\
\hline Giza178 & $1.50^{* \star}$ & $3.81^{* *}$ & $1.93^{* *}$ & $0.42^{* *}$ & $2.65^{\star \star}$ & $3.19^{\star \star}$ & $0.041^{\star *}$ & $0 . \check{-}^{-} 6^{* \star}$ & $0.118^{* *}$ & $0.042^{* *}$ & $0.224^{* *}$ & $0 . \overline{130^{* *}}$ & $0.180^{\star *}$ & $0.122^{* *}$ & $0.153^{* *}$ \\
\hline A22 & $0.84^{* *}$ & $3.01^{* *}$ & $1.88^{* *}$ & $0.09^{* *}$ & $1.77^{\star \star}$ & $1.73^{\star *}$ & $0.030^{\star \star}$ & $0 . \overline{-} 192^{\star \star}$ & $0 . \overline{-}$ & $0.022^{* *}$ & $0 . \overline{161^{\star \star}}$ & $0 . \overline{0} 0^{* *}$ & $0.160^{* *}$ & $0.079^{* *}$ & $0.164^{* *}$ \\
\hline IRAT170 & $0.32^{*}$ & $1.41^{* *}$ & $0.64^{* *}$ & $0.24^{* *}$ & $1.28^{* \star}$ & $0.13^{*}$ & $0.028^{\star *}$ & $0.204^{* *}$ & $0.039^{* *}$ & $0.038^{\star *}$ & $0.162^{* *}$ & $0.047^{\star *}$ & $0.097^{\star \star}$ & $0.094^{\star *}$ & $0.056^{* *}$ \\
\hline $\begin{array}{c}\text { WAB56- } \\
125 \\
\end{array}$ & $0.56^{* *}$ & $0.97^{\star *}$ & $1.65^{\star *}$ & $0.25^{\star \star}$ & $1.23^{\star \star}$ & $2.89^{\star \star}$ & $0.036^{* \star}$ & $0 . \overline{-} 5^{* \star}$ & $0 . \overline{-}$ & $0.047^{\star \star}$ & $0.085^{\star *}$ & $0.044^{* *}$ & $0.090^{\star *}$ & $0.074^{* *}$ & $0.106^{* *}$ \\
\hline S.E. (gi) & 0.22 & 0.14 & 0.21 & 0.04 & 0.10 & 0.12 & 0.003 & 0.002 & 0.005 & 0.004 & 0.004 & 0.006 & 0.010 & 0.002 & 0.008 \\
\hline S.E.(gi-gi) & 0.34 & 0.22 & 0.32 & 0.06 & 0.15 & 0.17 & 0.005 & 0.003 & 0.007 & 0.006 & 0.006 & 0.009 & 0.015 & 0.003 & 0.011 \\
\hline
\end{tabular}

${ }^{*}$ and ${ }^{* *}$ : Significant at 0.05 and 0.01 levels of probability, respectively, $\mathrm{N}=$ Normal environment,

$\mathrm{S}=$ salinity and $\mathrm{D}=$ Drought environment 
Table 7: Estimates of specific combining ability (SCA) effects for agronomic traits of 28 hybrids under normal, salinity and drought conditions

\begin{tabular}{|c|c|c|c|c|c|c|c|c|c|c|c|c|c|c|c|}
\hline \multirow[t]{2}{*}{ Hybrids } & \multicolumn{3}{|c|}{ Days to Heading (days) } & \multicolumn{3}{|c|}{ Plant height $(\mathrm{cm})$} & \multicolumn{3}{|c|}{ Panicles plant ${ }^{-1}$} & \multicolumn{3}{|c|}{ Filled grains panicle ${ }^{-1}$} & \multicolumn{3}{|c|}{ Grain yield plant ${ }^{-1}(\mathrm{~g})$} \\
\hline & $\mathrm{N}$ & $S$ & D & $\mathrm{N}$ & $\mathrm{S}$ & D & $\mathrm{N}$ & S & $\mathrm{D}$ & $\mathrm{N}$ & $\mathrm{S}$ & D & $\mathrm{N}$ & $\mathrm{s}$ & $\mathrm{D}$ \\
\hline Sakha102 X Sakha104 & $-3.28^{* *}$ & $-3.26^{* *}$ & $-5.83^{* \star}$ & $-6.02^{* *}$ & $8.06^{* *}$ & 1.24 & -1.24 & -0.97 & 0.14 & $-3.26^{* \star}$ & $4.21^{* *}$ & -0.30 & $-1.63^{*}$ & $-2.06^{* *}$ & -0.26 \\
\hline Sakha102 X Sakha105 & 0.65 & -0.26 & 0.81 & $-6.49^{* *}$ & $-7.21^{\star *}$ & -1.26 & $-3.37^{* *}$ & -0.34 & $-3.17^{* *}$ & $-6.63^{* *}$ & $-4.03^{* *}$ & -0.83 & $-9.61^{* *}$ & $-1.80^{* *}$ & $-3.29^{* *}$ \\
\hline Sakha102 X Sakha106 & $-2.15^{* *}$ & $-2.26^{*}$ & -0.49 & $-4.82^{* *}$ & $-5.38^{* *}$ & $-5.56^{\star \star}$ & -1.34 & -1.07 & -0.98 & $-1.87^{\star}$ & -0.49 & 0.43 & $-3.45^{* *}$ & $-2.42^{* *}$ & -1.24 \\
\hline Sakha102 X Giza178 & $2.05^{*}$ & 0.44 & 1.14 & $8.12^{* *}$ & $5.92^{* *}$ & $7.54^{* *}$ & $3.06^{* *}$ & $2.13^{* *}$ & $3.04^{* *}$ & -0.53 & $-1.73^{*}$ & $5.07^{* *}$ & $9.93^{* *}$ & $3.41^{* *}$ & 1.41 \\
\hline Sakha102 X A22 & $3.42^{* *}$ & $2.48^{*}$ & 1.34 & $8.55^{* *}$ & $13.32^{* *}$ & $5.94^{* *}$ & $2.69^{* *}$ & $1.56^{*}$ & $3.50^{* *}$ & 1.42 & $3.79^{* *}$ & 0.92 & $4.11^{* *}$ & $5.14^{* *}$ & $5.49^{* *}$ \\
\hline Sakha102 X IRAT170 & $2.92^{* *}$ & $3.78^{* *}$ & $3.97^{* *}$ & $5.02^{* *}$ & $-12.7^{* *}$ & $6.87^{* *}$ & $1.86^{*}$ & $-1.47^{*}$ & 1.04 & $-2.78^{* *}$ & -0.76 & 0.32 & $3.42^{* *}$ & $-3.31^{* *}$ & $2.19^{* *}$ \\
\hline Sakha102 X WAB56-125 & 0.02 & -0.22 & -1.09 & $8.42^{* *}$ & $8.39^{* *}$ & $6.21^{* *}$ & -0.57 & $1.96^{* *}$ & 0.04 & $10.08^{* *}$ & $3.72^{* *}$ & -0.42 & 0.21 & $4.93^{* *}$ & $2.98^{* *}$ \\
\hline Sakha104 X Sakha105 & $-2.82^{* *}$ & -1.16 & $-2.09^{* *}$ & $-4.79^{* *}$ & $-9.31^{* *}$ & $-9.19^{\star *}$ & -0.74 & -0.40 & 0.10 & $-2.36^{* *}$ & $-5.13^{\star \star}$ & $-8.13^{* *}$ & $-9.24^{* *}$ & $-3.00^{* *}$ & $-4.59^{* *}$ \\
\hline Sakha104 X Sakha106 & 1.05 & 1.51 & $3.27^{* *}$ & $-3.45^{* *}$ & $-7.48^{* *}$ & $-9.83^{\star *}$ & $-1.71^{*}$ & -1.14 & $-3.04^{* *}$ & $-13.31^{* \star}$ & $-6.69^{\star *}$ & $-9.50^{* *}$ & $-8.61^{* *}$ & $-4.13^{* *}$ & $-6.26^{* *}$ \\
\hline Sakha104 X Giza178 & 1.25 & 0.21 & $2.91^{* *}$ & $15.15^{* *}$ & $5.16^{* *}$ & $3.61^{* *}$ & $3.03^{* *}$ & $1.73^{*}$ & $1.97^{* *}$ & $-3.40^{* *}$ & $5.43^{* *}$ & 1.31 & $6.25^{* *}$ & $5.98^{* *}$ & $4.66^{* *}$ \\
\hline Sakha104 X A22 & $2.62^{* *}$ & $3.58^{* *}$ & $3.77^{* *}$ & $2.25^{*}$ & $6.22^{* *}$ & $7.01^{* *}$ & 0.33 & $2.83^{*}$ & 0.44 & $11.95^{* *}$ & $5.05^{* *}$ & $4.99^{* *}$ & -0.56 & $2.43^{* *}$ & 1.03 \\
\hline Sakha104 X IRAT170 & 1.45 & 1.88 & $1.74^{\star}$ & 0.38 & $5.82^{* *}$ & $4.94^{* *}$ & -0.17 & 0.46 & -1.36 & $9.35^{* *}$ & 0.76 & $5.02^{* *}$ & $2.85^{* *}$ & 0.02 & 1.46 \\
\hline Sakha104 X WAB56-125 & $2.55^{* *}$ & $2.21^{*}$ & $3.67^{* *}$ & $-2.89^{* *}$ & $5.62^{* *}$ & $3.61^{* *}$ & -0.94 & $1.56^{*}$ & 0.30 & $9.63^{* *}$ & $2.71^{* *}$ & $3.35^{* *}$ & $2.03^{* *}$ & $4.17^{* *}$ & $3.51^{* *}$ \\
\hline Sakha105 X Sakha106 & 1.32 & 1.51 & $1.91^{*}$ & $-10.9^{* *}$ & -1.74 & $-7.66^{* *}$ & 0.49 & -0.50 & -0.68 & $-7.38^{* *}$ & $-3.64^{* *}$ & -0.89 & $-5.42^{* *}$ & 0.44 & 0.76 \\
\hline Sakha105 X Giza178 & -0.82 & -1.46 & 0.54 & $5.35^{* *}$ & 1.56 & $7.44^{* *}$ & -0.11 & 0.36 & 1.00 & $-1.90^{*}$ & $-5.68^{\star *}$ & $-6.28^{* *}$ & -0.28 & $1.77^{\star \star *}$ & -0.29 \\
\hline Sakha105 X A22 & -1.12 & -0.76 & 0.41 & $16.78^{* *}$ & $12.62^{* *}$ & $15.17^{\star \star}$ & $2.53^{* *}$ & $2.13^{* *}$ & $4.46^{* *}$ & $2.06^{\star}$ & $-2.15^{* *}$ & 0.60 & $11.39^{* *}$ & $3.64^{* *}$ & $9.87^{* *}$ \\
\hline Sakha105 X IRAT170 & $5.05^{* *}$ & 1.88 & $1.71^{\star}$ & $-4.42^{* *}$ & $4.89^{* *}$ & -1.56 & $-1.97^{*}$ & 0.10 & -1.00 & $7.52^{* *}$ & $4.52^{* *}$ & $7.38^{* *}$ & $-3.97^{\star *}$ & -0.45 & -1.30 \\
\hline Sakha105 X WAB56-125 & $2.82^{* *}$ & $3.88^{* *}$ & $3.64^{* *}$ & $12.98^{* *}$ & $9.02^{* *}$ & $10.45^{\star \star}$ & $2.93^{* *}$ & 0.86 & 1.33 & 0.11 & -0.46 & $-3.29^{* *}$ & $12.68^{* *}$ & -0.97 & 0.78 \\
\hline Sakha106 X Giza178 & -1.28 & -0.46 & $1.57^{\star}$ & $4.68^{* *}$ & 1.06 & $8.47^{* *}$ & -0.41 & 0.30 & 0.86 & 0.99 & -0.70 & $-6.29^{* *}$ & $-2.91^{* *}$ & -0.66 & $-3.58^{* *}$ \\
\hline Sakha106 X A22 & -0.92 & 1.58 & -0.56 & $12.78^{* *}$ & $17.12^{\star *}$ & $15.21^{\star *}$ & 1.23 & $2.73^{* *}$ & $2.32^{* *}$ & 1.66 & 0.65 & 1.09 & $5.76^{* *}$ & $5.47^{* *}$ & $4.86^{* *}$ \\
\hline Sakha106 X IRAT170 & -1.08 & -1.46 & $-1.26^{*}$ & $-6.09^{* *}$ & $-12.3^{\star \star}$ & $2.47^{* *}$ & -0.27 & 0.03 & -0.81 & $4.40^{* *}$ & $1.80^{*}$ & $8.82^{* *}$ & $6.65^{* *}$ & -0.45 & 0.07 \\
\hline Sakha106 X WAB56-125 & $3.35^{* *}$ & 1.88 & $2.34^{* *}$ & $8.98^{* *}$ & $3.19^{* *}$ & $10.15^{\star *}$ & $-1.71^{*}$ & 0.80 & $1.52^{*}$ & -1.68 & $-3.37^{* *}$ & $-2.85^{* *}$ & $-2.81^{* *}$ & -0.23 & -0.38 \\
\hline Giza178 X A22 & -1.05 & $-4.72^{* *}$ & $-5.26^{* *}$ & $-10.3^{* *}$ & $-9.24^{* *}$ & $-11.7^{\star \star}$ & $-2.04^{*}$ & $-2.07^{* *}$ & $-5.66^{* *}$ & $16.81^{* *}$ & $3.99^{* *}$ & $-3.80^{* *}$ & -1.07 & $-6.77^{* *}$ & $-8.47^{* *}$ \\
\hline Giza178 X IRAT170 & $-2.88^{* *}$ & $-3.09^{* *}$ & $-6.63^{* *}$ & $4.85^{* *}$ & $5.02^{* *}$ & $2.57^{*}$ & -0.21 & $2.56^{* *}$ & 0.87 & $-9.05^{* *}$ & 0.08 & $-4.47^{* *}$ & $-3.02^{* *}$ & $2.77^{\star \star}$ & $5.56^{* *}$ \\
\hline Giza178 X WAB56-125 & -1.12 & 0.58 & $-3.36^{* \star}$ & $-6.42^{* *}$ & $-9.51^{\star *}$ & $-6.42^{\star *}$ & -0.64 & -0.67 & -0.46 & $4.81^{* *}$ & $9.86^{* *}$ & $10.37^{\star \star}$ & $1.75^{*}$ & 0.46 & $2.13^{* *}$ \\
\hline A22 X IRAT170 & -0.85 & 1.61 & $1.91^{\star}$ & $6.28^{* *}$ & 0.76 & $-4.03^{* *}$ & 1.43 & 1.00 & $-1.66^{*}$ & $-10.50^{* \star}$ & $-7.58^{* *}$ & $-7.69^{\star \star}$ & $1.93^{* *}$ & -0.65 & $-3.91^{* *}$ \\
\hline A22 X WAB56-125 & -0.08 & -0.39 & -1.49 & $-11.3^{* *}$ & $-19.1^{\star *}$ & $-7.02^{\star *}$ & $1.99^{*}$ & $-1.57^{*}$ & $-3.00^{* *}$ & $-1.95^{\star}$ & $5.23^{* *}$ & $10.08^{\star \star}$ & $2.82^{* *}$ & $-3.43^{* *}$ & $-3.89^{* *}$ \\
\hline IRAT170 X WAB56-125 & $-2.58^{* *}$ & $-2.42^{*}$ & 0.14 & -0.85 & $9.82^{* *}$ & -0.42 & -1.17 & 0.73 & $1.87^{* *}$ & $-12.36^{* \star}$ & -0.85 & $-9.96^{* *}$ & $-5.19^{* *}$ & $1.86^{* *}$ & -0.60 \\
\hline LSD at 0.05 & 1.56 & 2.02 & 1.53 & 1.99 & 1.93 & 2.19 & 1.63 & 1.39 & 1.38 & 1.72 & 1.58 & 1.81 & 1.39 & 1.27 & 1.48 \\
\hline LSD at 0.01 & 2.10 & 2.73 & 2.07 & 2.68 & 2.61 & 2.95 & 2.20 & 1.87 & 1.86 & 2.32 & 2.12 & 2.45 & 1.88 & 1.71 & 1.99 \\
\hline
\end{tabular}


Table 7: continue.....

\begin{tabular}{|c|c|c|c|c|c|c|c|c|c|c|c|c|c|c|c|}
\hline \multirow{2}{*}{ Hybrids } & \multicolumn{3}{|c|}{ Total chlorophyll content (SPAD) } & \multicolumn{3}{|c|}{ Proline content $(\mu \mathrm{g} / \mathrm{g})$} & \multicolumn{3}{|c|}{$\mathrm{Na}^{+} / \mathrm{k}^{+}$ratio } & \multicolumn{3}{|c|}{$\mathrm{Na}^{+}$content $(\%)$} & \multicolumn{3}{|c|}{$\mathrm{K}^{+}$content $(\%)$} \\
\hline & $\mathrm{N}$ & $\mathrm{S}$ & $\mathrm{D}$ & $\mathrm{N}$ & $\mathrm{S}$ & $\mathrm{D}$ & $\mathrm{N}$ & $\mathrm{S}$ & $\mathrm{D}$ & $\mathrm{N}$ & $\mathrm{S}$ & $\mathrm{D}$ & $\mathrm{N}$ & $S$ & $\mathrm{D}$ \\
\hline Sakha102 X Sakha104 & $3.39^{* *}$ & -0.46 & -0.79 & -0.042 & $-1.82^{* *}$ & $-1.22^{*}$ & 0.033 & -0.108 & 0.065 & 0.040 & -0.025 & 0.030 & -0.116 & 0.091 & -0.127 \\
\hline Sakha102 X Sakha105 & $-3.87^{\star *}$ & $-4.22^{\star *}$ & $-2.01^{*}$ & -0.033 & $-1.55^{\star \star}$ & $-3.61^{* *}$ & 0.044 & -0.080 & 0.090 & 0.058 & 0.013 & 0.111 & -0.148 & 0.078 & -0.049 \\
\hline Sakha102 X Sakha106 & $-4.59^{* *}$ & $-1.47^{*}$ & $-4.43^{* *}$ & 0.531 & -0.34 & $-2.40^{* *}$ & -0.025 & $-0.174^{*}$ & 0.053 & -0.030 & -0.154 & 0.056 & 0.026 & 0.055 & -0.047 \\
\hline Sakha102 X Giza178 & $3.96^{* *}$ & $3.83^{* *}$ & $3.63^{* *}$ & 0.055 & 0.95 & $-2.50^{* \star}$ & -0.059 & 0.033 & -0.099 & -0.069 & 0.094 & -0.136 & $0.360^{*}$ & 0.020 & 0.065 \\
\hline Sakha102 X A22 & 1.32 & $3.62^{* *}$ & $2.98^{* \star}$ & 0.577 & $-1.47^{*}$ & $4.05^{\star \star}$ & -0.030 & 0.015 & -0.008 & -0.034 & 0.048 & -0.047 & 0.134 & 0.018 & -0.097 \\
\hline Sakha102 X IRAT170 & -0.70 & $2.55^{\star \star}$ & -0.08 & -0.258 & -0.98 & $2.42^{* \star}$ & -0.022 & 0.079 & -0.123 & -0.032 & 0.171 & -0.084 & 0.016 & 0.042 & 0.221 \\
\hline Sakha102 X WAB56-125 & 0.19 & $2.99^{* *}$ & 1.24 & -0.309 & $2.98^{* *}$ & -0.04 & -0.039 & $-0.249^{* \star}$ & -0.118 & -0.041 & $-0.364^{* *}$ & -0.161 & 0.205 & -0.018 & 0.080 \\
\hline Sakha104 X Sakha105 & $1.82^{*}$ & $2.47^{* \star}$ & 0.48 & -0.211 & $1.21^{*}$ & 0.29 & 0.043 & -0.087 & 0.026 & 0.119 & -0.148 & 0.069 & 0.114 & -0.031 & 0.036 \\
\hline Sakha104 X Sakha106 & $-3.82^{* *}$ & $-2.17^{* *}$ & -0.14 & $-0.781^{*}$ & -0.51 & $-4.37^{* *}$ & 0.025 & 0.138 & 0.057 & 0.061 & $0.286^{*}$ & 0.104 & 0.075 & 0.055 & 0.019 \\
\hline Sakha104 X Giza178 & $-3.23^{\star *}$ & $-1.68^{*}$ & $-3.59^{* *}$ & 0.110 & -0.79 & $5.46^{* *}$ & -0.041 & $-0.275^{* *}$ & -0.071 & -0.083 & $-0.362^{* \star}$ & -0.090 & -0.028 & 0.057 & 0.066 \\
\hline Sakha104 X A22 & $-6.31^{* *}$ & $-2.69^{* *}$ & $-2.94^{* *}$ & 0.289 & -1.01 & 0.18 & -0.038 & -0.001 & 0.029 & -0.082 & 0.029 & 0.056 & -0.048 & 0.025 & -0.011 \\
\hline Sakha104 X IRAT170 & 1.01 & -0.53 & 2.01 & 0.297 & $1.24^{*}$ & $3.71^{* *}$ & 0.003 & $0.225^{\star *}$ & 0.033 & 0.016 & 0.116 & 0.031 & 0.062 & -0.108 & -0.015 \\
\hline Sakha104 XWAB56-125 & $-3.77^{* *}$ & -1.35 & -0.98 & 0.379 & $1.54^{* *}$ & $-2.14^{* *}$ & -0.073 & 0.155 & -0.039 & -0.154 & 0.173 & -0.056 & $\frac{0.006}{-0.073}$ & -0.057 & 0.008 \\
\hline Sakha105 X Sakha106 & -0.41 & -0.20 & $-1.69^{*}$ & -0.239 & $-1.94^{* *}$ & $-3.85^{* \star}$ & 0.004 & 0.082 & 0.155 & -0.053 & -0.051 & 0.155 & -0.131 & -0.093 & -0.080 \\
\hline Sakha105 X Giza178 & $1.48^{\star}$ & $3.39^{* *}$ & $3.09^{* *}$ & $0.785^{*}$ & $3.19^{* *}$ & $4.64^{* *}$ & -0.075 & -0.075 & -0.076 & -0.098 & -0.101 & -0.097 & 0.181 & -0.036 & -0.007 \\
\hline Sakha105 X A22 & $3.10^{* \star}$ & $3.19^{* *}$ & $2.71^{* *}$ & 0.408 & $3.00^{* *}$ & $3.93^{* *}$ & -0.059 & $-0.191^{*}$ & -0.071 & -0.041 & $-0.280^{*}$ & -0.012 & 0.295 & -0.028 & 0.165 \\
\hline Sakha105 X IRAT170 & -0.44 & $-3.56^{* *}$ & $2.25^{\star *}$ & 0.006 & $2.65^{* *}$ & 0.83 & -0.012 & $0.186^{*}$ & 0.009 & -0.017 & $0.309^{* *}$ & -0.010 & 0.012 & 0.048 & -0.024 \\
\hline Sakha105 X WAB56-125 & 1.15 & $3.59^{* *}$ & $2.64^{* *}$ & -0.444 & 0.68 & 0.24 & -0.014 & $-0.195^{\star}$ & -0.001 & -0.041 & -0.105 & -0.001 & -0.134 & 0.114 & -0.046 \\
\hline Sakha106 X Giza178 & $3.39^{* *}$ & $2.51^{* *}$ & $3.34^{* *}$ & 0.415 & $4.56^{* *}$ & $5.15^{* *}$ & 0.013 & 0.024 & -0.098 & 0.046 & 0.016 & -0.109 & 0.020 & -0.051 & 0.079 \\
\hline Sakha106 X A22 & 1.54 & 1.81 & $3.83^{\star \star}$ & -0.010 & $1.57^{* \star}$ & $5.34^{\star *}$ & 0.020 & $-0.167^{*}$ & -0.062 & 0.014 & -0.043 & -0.139 & -0.139 & $0.170^{*}$ & -0.110 \\
\hline Sakha106 X IRAT170 & 0.33 & -0.17 & 1.33 & -0.381 & $1.29^{*}$ & $1.87^{\star \star}$ & -0.003 & $0.291^{* *}$ & 0.001 & 0.051 & $0.289^{*}$ & 0.046 & 0.215 & -0.040 & 0.074 \\
\hline Sakha106 X WAB56-125 & 1.49 & $2.71^{* *}$ & $3.25^{* *}$ & 0.351 & 0.92 & 1.18 & 0.003 & -0.012 & -0.016 & -0.018 & -0.021 & 0.025 & -0.120 & -0.031 & 0.056 \\
\hline Giza178 X A22 & -0.70 & 0.93 & $1.71^{*}$ & -0.705 & $-1.87^{\star \star}$ & $-6.67^{\star *}$ & 0.003 & 0.144 & 0.060 & -0.005 & 0.147 & 0.123 & -0.023 & -0.036 & 0.084 \\
\hline Giza178 X IRAT170 & $-2.25^{*}$ & $2.22^{* \star}$ & -0.99 & -0.006 & $-1.18^{*}$ & $1.76^{\star \star}$ & 0.049 & -0.142 & 0.135 & 0.021 & $-0.218^{*}$ & 0.115 & -0.318 & -0.059 & -0.241 \\
\hline Giza178 X WAB56-125 & $-2.26^{*}$ & -0.20 & -1.57 & -0.358 & $-2.42^{\star *}$ & $-3.19^{* *}$ & 0.030 & -0.056 & 0.071 & 0.083 & 0.054 & 0.109 & 0.114 & $0.198^{\star *}$ & -0.032 \\
\hline A22 X IRAT170 & $-2.29^{* \star}$ & $1.55^{*}$ & -0.40 & -0.184 & $-2.14^{\star *}$ & $-4.21^{* \star}$ & 0.010 & $-0.224^{* \star}$ & 0.005 & 0.021 & -0.192 & 0.083 & -0.021 & 0.090 & 0.131 \\
\hline A22 X WAB56-125 & 0.56 & $1.60^{*}$ & -0.48 & -0.076 & $3.29^{* *}$ & $5.53^{\star \star}$ & -0.010 & 0.114 & 0.012 & 0.021 & 0.094 & 0.074 & 0.299 & -0.056 & 0.174 \\
\hline IRAT170 X WAB56-125 & 0.75 & -1.08 & -0.27 & 0.363 & $-3.16^{* \star}$ & $-1.34^{*}$ & -0.009 & $0.218^{* *}$ & 0.070 & -0.011 & 0.179 & -0.003 & 0.023 & -0.107 & -0.225 \\
\hline LSD at 0.05 & 1.69 & 1.36 & 1.64 & 0.717 & 1.13 & 1.22 & 0.200 & 0.161 & 0.243 & 0.231 & 0.217 & 0.281 & 0.356 & 0.146 & 0.311 \\
\hline LSD at 0.01 & 2.29 & 1.83 & 2.21 & 0.968 & 1.52 & 1.64 & 0.270 & 0.218 & 0.328 & 0.312 & 0.292 & 0.379 & 0.481 & 0.197 & 0.420 \\
\hline
\end{tabular}

${ }^{*}$ and ${ }^{* *}$ : Significant at 0.05 and 0.01 levels of probability, respectively, $\mathrm{N}=$ Normal environment,

$\mathrm{S}=$ salinity and $\mathrm{D}=$ Drought environment.

\section{Genetic diversity analysis of the tested rice varieties using SSR markers}

The eight parents used in the present study were subjected to DNA polymorphism screening and assessment using SSR markers which offer a great potential for generating large numbers of markers evenly distributed throughout the genome and have efficiently been used to give reliable and reproducible genetic markers. Eight SSR primer pairs related to salinity and drought tolerance with known map positions distributed in the rice genome were used to screen a set of eight selected Indica, Japonica and Tropical-Japonica rice genotypes with different levels and mechanisms of drought and salinity tolerance. The presence, absence matrix for SSR amplified fragments for the studied genotypes are found in Table (8).

\section{Number of alleles and allelic diversity}

The eight SSR markers spread on four chromosomes (1, 6, 8 and 9) generated polymorphic alleles. Data in Table (8) showed that, a total number of 29 alleles were detected at the loci of the eight markers across the eight rice genotypes. The number of alleles per locus generated by each marker varied from 2(RM201) to 5 (RM223 and RM25) alleles with an average of 3.63 alleles per locus. Only one SSR 
marker generated two alleles, three markers generated three alleles, two markers generated four alleles and two generated five alleles. The eight primers gave a total of 29 DNA fragments from all tested varieties with an average of 3.63 bands per primer. The varieties Sakha104, Sakha106, Giza178 and A22 displayed the highest number of DNA fragments (13 fragments), while variety IRAT170 revealed the lowest number of fragments (8). Data in Table (9) showed that, the effective number of alleles per locus ranged from 1.60 alleles to 4.92 alleles with an average of 3.26 alleles. The highest and effective numbers of alleles per locus was observed for RM72 (4.92), RM3805 (4.74) and RM223 (4.44). Results in Table (9) showed also that the lowest numbers of alleles per locus were obtained by RM201 (1.60) and RM315 (2.13) similar results were obtained by Sajib et al., 2012 (3.33) and Vanniarajan et al., 2012 (2.5). On the other hand, high number of alleles per locus was obtained by El-Malky et al. 2007 (8.57); Ni et al. 2002 (6.8) and Ram et al. 2007 (4.86). On average, 45\% of the eight rice genotypes shared common major allele at any given locus ranging from 31\% (RM219, RM72 and RM223) and 75\% (RM201).

Table 8: The presence (1), absence (0) matrix for SSR amplified fragments for the studied genotypes

\begin{tabular}{|c|c|c|c|c|c|c|c|c|c|c|}
\hline Marker & $\begin{array}{l}\text { No. of } \\
\text { alleles. }\end{array}$ & $\begin{array}{c}\text { Sakha } \\
102\end{array}$ & $\begin{array}{c}\text { Sakha } \\
104\end{array}$ & $\begin{array}{c}\text { Sakha } \\
105\end{array}$ & $\begin{array}{c}\text { Sakha } \\
106\end{array}$ & $\begin{array}{c}\text { Giza } \\
178\end{array}$ & A22 & $\begin{array}{c}\text { IRAT } \\
170\end{array}$ & $\begin{array}{c}\text { WAB } \\
56-125\end{array}$ & $\begin{array}{l}\text { M.W. } \\
\text { (bp) }\end{array}$ \\
\hline \multirow{4}{*}{ RM 219} & 1 & 1 & 1 & 1 & 1 & 0 & 0 & 0 & 0 & 600 \\
\hline & 2 & 0 & 1 & 0 & 0 & 1 & 0 & 0 & 0 & 500 \\
\hline & 3 & 1 & 0 & 1 & 1 & 0 & 1 & 0 & 0 & 250 \\
\hline & 4 & 0 & 0 & 0 & 0 & 0 & 0 & 1 & 1 & 200 \\
\hline \multirow{2}{*}{ RM 201} & 1 & 1 & 1 & 1 & 1 & 1 & 1 & 0 & 0 & 150 \\
\hline & 2 & 0 & 0 & 0 & 0 & 0 & 0 & 1 & 1 & 100 \\
\hline \multirow{3}{*}{ RM 3805} & 1 & 1 & 1 & 0 & 1 & 0 & 0 & 0 & 0 & 125 \\
\hline & 2 & 0 & 0 & 0 & 0 & 1 & 1 & 0 & 1 & 75 \\
\hline & 3 & 1 & 1 & 0 & 1 & 1 & 1 & 0 & 1 & 50 \\
\hline \multirow{4}{*}{ RM 72} & 1 & 1 & 1 & 1 & 1 & 0 & 0 & 0 & 0 & 200 \\
\hline & 2 & 0 & 0 & 0 & 0 & 1 & 0 & 0 & 0 & 125 \\
\hline & 3 & 0 & 0 & 0 & 0 & 0 & 1 & 0 & 0 & 100 \\
\hline & 4 & 0 & 1 & 1 & 1 & 1 & 1 & 0 & 0 & 50 \\
\hline \multirow{5}{*}{ RM 223} & 1 & 1 & 1 & 0 & 1 & 0 & 0 & 0 & 0 & 400 \\
\hline & 2 & 0 & 0 & 0 & 0 & 1 & 1 & 0 & 1 & 370 \\
\hline & 3 & 0 & 0 & 0 & 0 & 1 & 1 & 0 & 1 & 350 \\
\hline & 4 & 0 & 0 & 0 & 0 & 1 & 1 & 1 & 1 & 200 \\
\hline & 5 & 1 & 1 & 1 & 1 & 0 & 0 & 0 & 0 & 150 \\
\hline \multirow{3}{*}{ RM 315} & 1 & 1 & 1 & 1 & 1 & 0 & 0 & 1 & 0 & 150 \\
\hline & 2 & 0 & 0 & 0 & 0 & 1 & 1 & 0 & 0 & 140 \\
\hline & 3 & 0 & 0 & 0 & 0 & 0 & 0 & 0 & 1 & 125 \\
\hline \multirow{3}{*}{ RM 8094} & 1 & 1 & 1 & 1 & 1 & 1 & 1 & 0 & 0 & 500 \\
\hline & 2 & 1 & 1 & 1 & 1 & 0 & 1 & 0 & 0 & 250 \\
\hline & 3 & 0 & 0 & 0 & 0 & 1 & 0 & 1 & 0 & 200 \\
\hline \multirow{5}{*}{ RM 25} & 1 & 0 & 0 & 0 & 0 & 0 & 0 & 1 & 0 & 230 \\
\hline & 2 & 0 & 0 & 0 & 0 & 0 & 0 & 1 & 0 & 210 \\
\hline & 3 & 0 & 0 & 0 & 0 & 0 & 0 & 1 & 0 & 190 \\
\hline & 4 & 0 & 0 & 0 & 1 & 1 & 1 & 0 & 1 & 150 \\
\hline & 5 & 1 & 1 & 0 & 0 & 0 & 0 & 0 & 0 & 130 \\
\hline \multicolumn{2}{|c|}{ Total band } & 12 & 13 & 9 & 13 & 13 & 13 & 8 & 9 & \\
\hline
\end{tabular}

A total of 29 amplified fragments were polymorphic (Table 9). The monomorphic fragments are constant and cannot be used to study the diversity while polymorphic fragments revealed differences and could be used to examine and establish systematic relationships among the genotypes (Hadrys et al. 1992). Results presented in Table 
(9) showed that the total number of polymorphic fragments was 29 bands, out of them six were unique, and the highest unique bands number was detected by primer RM25 with three unique bands. On the other hand, two unique fragments were scored by RM72 and one unique band was scored by RM315. Unique DNA fragments with different sizes were detected in a particular genotype but not in the others using different primers. The presence of a unique fragment for a given genotype is referred as positive marker, while the absence of common fragments served as negative marker. Such bands could be used as DNA markers for genotype identification and discrimination. In this respect, three DNA unique fragments were detected in the varieties, IRAT170 and one fragment in each of the genotypes Giza178, A22 and WAB56-125.

Table 9: List of SSR markers used including name, polymorphic bands (PM), unique bands (UN), number of amplified alleles (AN), effective number of alleles (ENA), common alleles (CA) and polymorphic information content (PIC)

\begin{tabular}{|c|c|c|c|c|c|c|c|}
\hline No. & Marker & PIC & ENA & AN & CA & UN. & PM \\
\hline 1 & RM 219 & 0.74 & 3.88 & 4 & 0.31 & 0 & $100 \%$ \\
\hline 2 & RM 201 & 0.38 & 1.60 & 2 & 0.75 & 0 & $100 \%$ \\
\hline 3 & RM 3805 & 0.79 & 4.74 & 3 & 0.38 & 0 & $100 \%$ \\
\hline 4 & RM 72 & 0.80 & 4.92 & 4 & 0.31 & 2 & $100 \%$ \\
\hline 5 & RM 223 & 0.78 & 4.44 & 5 & 0.31 & 0 & $100 \%$ \\
\hline 6 & RM 315 & 0.53 & 2.13 & 3 & 0.63 & 1 & $100 \%$ \\
\hline 7 & RM 8094 & 0.73 & 3.66 & 3 & 0.38 & 0 & $100 \%$ \\
\hline 8 & RM 25 & 0.68 & 3.15 & 5 & 0.50 & 3 & $100 \%$ \\
\hline
\end{tabular}

\section{PIC value:-}

PIC value refers to the value of a marker for detecting polymorphism within a population, depending on the number of detectable alleles and the distribution of their frequency; thus, it provides an estimate of the discriminating power of the marker (Nagy et al., 2012). As it is shown in Table (9), the PIC values for the SSR used in this study varied from 0.38 to 0.80 with an average of 0.68 . This result is consistent with that at Sajib et al. (2012) who reported greatly variations in PIC values for all tested SSR loci (from 0.14 to 0.71 with an average of 0.48 ). Higher averages of PIC values (0.57) were reported by Zeng et al. (2004) and (0.707) by Ram et al., (2007). According to Anderson et al. (1993), there were seven highly informative markers (PIC > 0.50), one informative marker $(50<\mathrm{PIC}<$ $0.25)$ and no slightly informative markers $(\mathrm{PIC}<0.25)$. The highest PIC values were observed for RM72 (0.80), RM3805 (0.79) and RM223 (0.78). 


\section{Genetic similarity and phylogenetic tree based on the SSR markers.}

Data in Table (10) showed that, the similarity matrices resulting from the SSR markers. In the present investigation, eight SSR markers were used to study the genetic differences and relationships among the eight rice varieties (Fig. 1). Polymorphism was evident in all tested SSR markers, polymorphism due to SSR markers analysis could be caused by differences in nucleotide sequences at the priming sites (such as point mutations), or by structural rearrangements within the amplified sequence, (e.g., insertions, deletions, inversions) (Welsh and McClelland, 1990).

The genetic similarity among the eight rice varieties was ranging from $0 \%$ to $79 \%$. The highest similarity $79 \%$ and shortest genetic distance were scored between Sakha102 with each of Sakha104 and Sakha106; these three varieties are Japonica rice. Moreover, the obtained results confirmed the sensitivity and moderately tolerant of those varieties to salt and drought stresses. The lowest genetic similarity $(0 \%)$ and longest genetic distance were found between WAB56-125 and Sakha105. These results were substantiated by the fact that these two genotypes have different origin, the genotypes WAB56-125 is Indica rice while, Sakha105 is Japonica rice, which may be due to the absence of common parents between them. Also, the large distance between Sakha105 and WAB56-125 fixed their difference in the ability of drought and salt tolerance. These results were in agreement with those of Chakravarthi and Naravaneni (2006) who reported that low similarity coefficient between Japonica type and Indica type genotypes were found, and Kanawapee et al. (2011) who reported relatively high level of similarity between closely related genotypes.

Table (10): Genetic similarity and distance values of SSR markers among the eight rice varieties

\begin{tabular}{|l|l|l|l|l|l|l|l|}
\hline & $\begin{array}{l}\text { Sakha } \\
102\end{array}$ & $\begin{array}{l}\text { Sakha } \\
104\end{array}$ & $\begin{array}{l}\text { Sakha } \\
105\end{array}$ & $\begin{array}{l}\text { Sakha } \\
106\end{array}$ & $\begin{array}{l}\text { Giza } \\
178\end{array}$ & A22 & $\begin{array}{l}\text { IRAT } \\
170\end{array}$ \\
\hline Sakha102 & & & & & & & \\
\hline Sakha104 & 0.79 & & & & & & \\
\hline Sakha105 & 0.62 & 0.57 & & & & & \\
\hline Sakha106 & 0.79 & 0.73 & 0.69 & & & & \\
\hline Giza178 & 0.14 & 0.24 & 0.16 & 0.24 & & & \\
\hline A22 & 0.25 & 0.24 & 0.29 & 0.37 & 0.63 & & \\
\hline IRAT170 & 0.05 & 0.05 & 0.06 & 0.05 & 0.11 & 0.05 & \\
\hline WAB56-125 & 0.05 & 0.05 & 0.00 & 0.10 & 0.38 & 0.38 & 0.21 \\
\hline
\end{tabular}




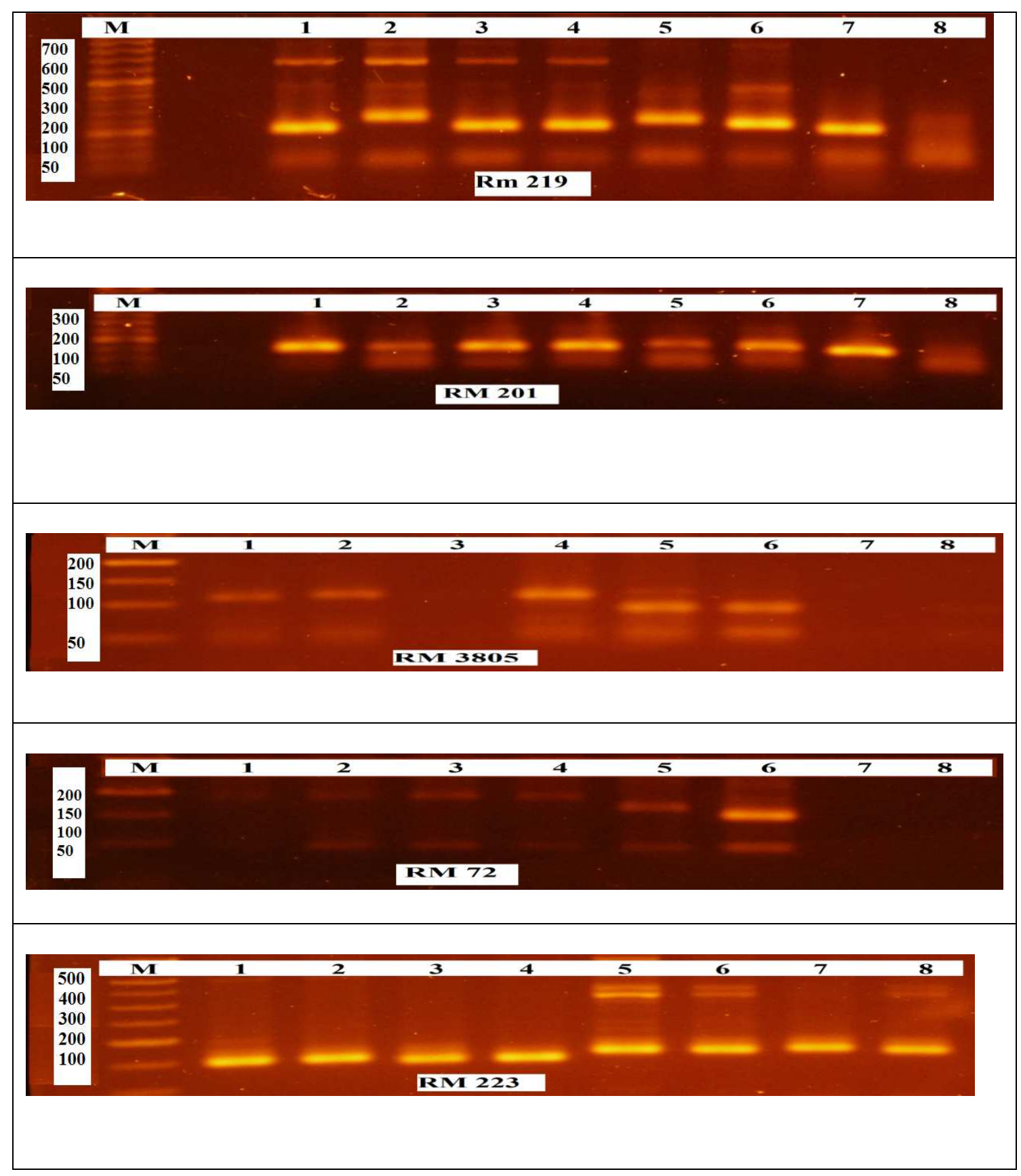




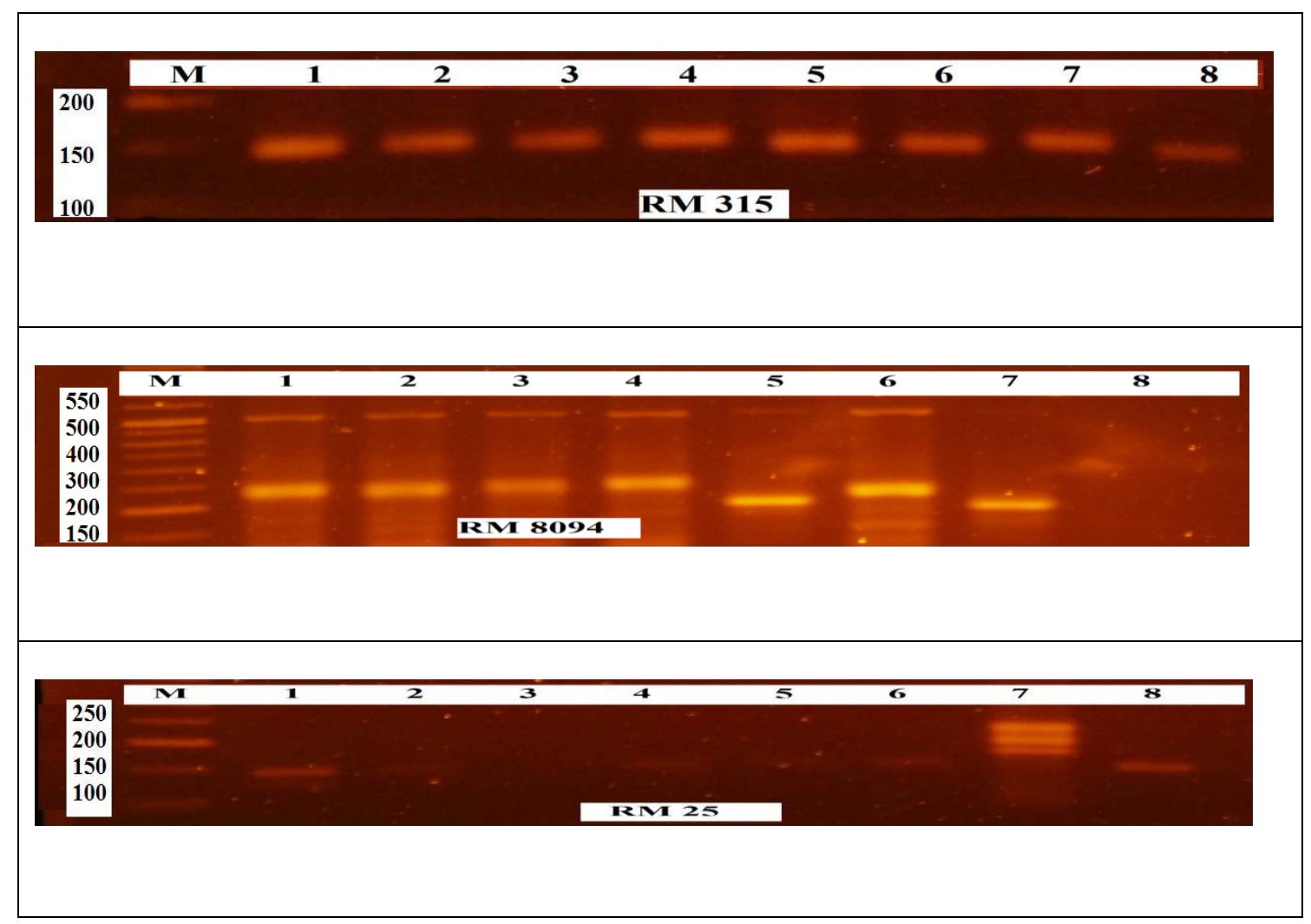

Fig. 1: Agarose gel electrophoresis of PCR amplified fragments for eight SSR markers. M is 50 bp DNA ladder; 1, Sakha102; 2, Sakha104; 3, Sakha105; 4, Sakha106; 5, Giza178 6, A22; 7, IRAT170; and 8, WAB56-125

\section{Cluster analysis:-}

The genetic relationships among rice genotypes are presented in a dendrogram based on informative microsatellite alleles (Fig. 2). All genotypes clearly grouped into two major clusters in the dendrogram at $9 \%$ similarity based on Jaccard's similarity index. The first cluster represents the tropical Japonica rice IRAT170. While, the second cluster represents two sup clusters, the first sub cluster include the Indica and Indica/Japonica rice Giza178, A22 and WAB56-125 at 38\% similarity based on Jaccard's similarity index and the second cluster include Japonica rice Sakha102, Sakha104, Sakha105 and Sakha106 at $62 \%$ similarity based on Jaccard's similarity index. Below the sub cluster Indica and Indica-Japonica in the dendrogram, Giza178 and A22 were in one group at $62 \%$ similarity and WAB56-125 was in another group at $38 \%$ similarity with other indica rice in the same sub 
cluster. Below the second sub cluster in the dendrogram, genotypes were grouped into three groups, the first group contains Sakha105, the second group contains Sakha106, and the third group contains Sakha102 and Sakha104 at about 62, 74 and $79 \%$ similarity. A seen in cluster result, IRAT170 came in first cluster since it was found to be only drought tolerance and salt sensitive. It was observed that, the four Japonica rice varieties; Sakha102, Sakha104, Sakha105 and Sakha106 occupied on the second sup cluster while all of them drought and salt sensitivity, except Sakha104 was moderately tolerance for salinity and drought stresses. Continuously, A22, Giza178 and WAB56-125 were in the first sup cluster and were characterized as drought and salt tolerance varieties. El-Malky et al. (2007) reported the ability of SSR makers to divide the genotypes into two groups, one included the Indica genotypes and the other included the Japonica genotypes. Also, Zeng et al. (2004) found that, all genotypes clearly grouped into two major branches in the dendrogram with less than $10 \%$ similarity based on Jaccard similarity index, one branch represented the subspecies Japonica rice and the other branch represented the subspecies Indica or the hybrids between Japonica rice and Indica rice.

\section{Identified MAS marker:-}

Among eight polymorphic SSR markers, RM223 was able to divide the studied genotypes into two groups depending on their drought tolerance (Figure 1). The first group showed the first allele with molecular size of $150 \mathrm{bp}$ included the drought susceptible genotypes i.e. Sakha102, Sakha104, Sakha105 and Sakha106. Where the second allele with molecular size of $200 \mathrm{bp}$ appeared in the second group included the drought tolerant genotypes i.e. Giza178, A22, IRAT170 and WAB56-125. Furthermore, this marker indicated that the three parent i.e. Giza178, A22 and WAB56-125 exhibited two allele differed with other parent this molecular result indicated that this three parent tolerant to salinity stress and this result supported agronomic and yield characters under this study, so this marker can used in breeding program under salinity and drought stress. These results agree with Lang et al. (2008) they used RM223 for salinity tolerance. Furthermore, Kumar et al. (2005) used this primer for drought tolerance in rice. 


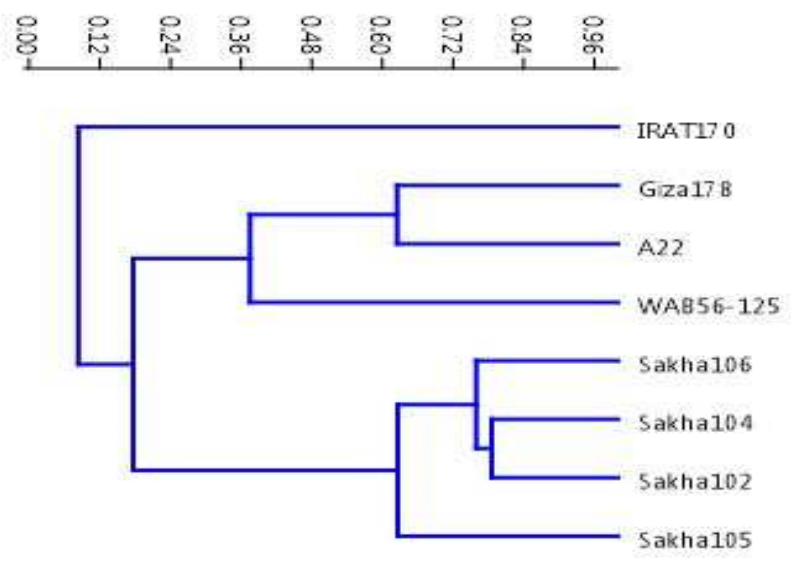

Figure 2: Dendrogram derived from UPGMA cluster analysis of eight rice genotypes based on Jaccard's similarity coefficient using eight SSR markers

\section{REFERENCE}

Anderson, J.A.; G.A. Churchill; J.E. Autrique; S.D. Tanksley and M.E. Sorrells(1993). Optimizing parental selection for genetic linkage maps. Genome, 36 (1):181-186.

Araus, J.L.; G.A. Slafer; M.P. Reynolds and C. Royo(2002). Plant breeding and relations in C3 cereals: what to breed for? Annals of Botany, 89: 925- 940.

Bastawisi, A.O.; H.F. El-Mowafi; M.A. Maximos and M.F. Sabaa(2003). Hybrid rice production technology in Egypt. Proceedings: Workshop on rice integrated crop management systems for food security in the near east countries, 27-29 July, Alexandria, Egypt.

Black, C.D.; D.D. Evans; L.E. Ensminger; J.L. White and F.E. Clark(1965). Methods of soil analysis. Part I and II. Amer. Soc. of Agron. in Publisher, Madison-Wisconsin, USA.

Butany, W.T.(1961). Mass emasculation in rice. International Rice Commission Newsletter, 9: 9-13.

Chaitra, J.; M.S. Vinod; N. Sharma; S. Hittalmani and H.E. Shashidhar(2006). Validation of markers linked to maximum root length in rice (Oryza sativa, L.). Current Science, 90(6): 835-838.

Chakravarthi, B.K. and R. Naravaneni(2006). SSR marker based DNA fingerprinting and diversity study in rice (Oryza sativa, L.). African Journal of Biotechnology, 5(9): 684-688.

Chapman, H.D. and F.P. Parker(1961). Methods of analysis for soils, plants and waters. Univ. of Calif. Division of Agricultural Sciences. 
Chatterjee, M. and P.J.M. Reddy(2014). Identification of salt tolerance genotypes using SSR markers in rice. Research Journal of Pharmaceutical, Biological and Chemical Sciences; 2014. 5(4):1312-1317. 12 ref.

El-Malky, M.M.; A.I. Fahmy and A.A. Kotb(2007). Detection of genetic diversity using microsatellites in rice (Oryza sative, L.). African Crop Science Conference Proceeding, 8: 597-603.

El-Mouhamady, A.A.; I.S. El-Demardash and K.A. Aboud(2010). Biochemical and Molecular Genetic Studies on Rice Tolerance to Salinity. Journal of American Science 2010;6(11).521-535.

El-Mowafi, H.F. and A.A. Abou Shousha(2003). Combining ability and heterosis analysis of divers CMS lines in hybrid rice. J. Agric. Res. Tanta Univ. 29(1): 106-127

Eynard, A.; R. Lal and K. Wiebe(2005). Crop response in salt-affected soils. J. Sustain.Agric. 27 (1), 5-50.

[FAO] Food and Agriculture Organization of the United Nations(2004). The state of food and agriculture 2003-2004. Agricultural Biotechnology: Meeting the Needs of the Poor?

Gracia, A.A.F; L.L. Benchimol; M.M. Antonica; I.O. Geraldi and A.P. Deuza(2004). Comparison of RAPD, RFLP, AFLP and SSR marker for diversity 145 studies in tropical maize inbred lines. Euphytica, 108: 53-63.

Griffing, B.(1956). Concept of general and specific combining ability in relation to diallel hybriding systems. Australian Journal of Biological Sciences, 9: 463-493.

Hadrys, H.; M. Balick and B. Schiewater(1992). Application of random amplified polymorphic DNA (RAPD) in molecular ecology. Mol. Ecol., 1: 55-60.

Hammer, Ø; D.A.T. Harper and P.D. Ryan(2001). PAST: Paleontological statistics software package for education and data analysis. Palaeontologia Electronica, 4(1): 1-9.

Hammoud, S.A.A.; I.S.M. EL-Degwy; S.E.M. Sedeek and B.A. Zayed(2008). Line $X$ tester analysis for some rice quantitative traits.2nd Field Crops Conference 14-16 Oct.,Giza, Egypt 121-140.

Jodon, N. E.(1938). Experiments on artificial hybridization of rice. Journal of the American Society of Agronomy, 30 (4): 294-305.

Kanawapee, N.; J. Sanitchon; P. Srihaban and P. Theerakulpisut(2011). Genetic diversity analysis of rice cultivars (Oryza sativa, L.) differing in salinity tolerance based on RAPD and SSR markers. Electronic Journal of Biotechnology, 14(6): 1-17.

Kumar, B.; S.M. Gomez; N.M. Boopathi; S.S. Kumar; D. Kumaresan; K.R. Biji; B.K. Babu; N.S.R. Prasad; P. Shanmugasundaram and R.C. Babu(2005). Identification of Microsatellite Markers Associated with Drought Tolerance in Rice (Oryza sativa L.) using Bulked Line Analysis. Tropical Agricultural Research Vol. 17:39-47.

Lang, N.T.; B.C. Buu and A. Ismail(2008). molecular mapping and marker-assisted selection for salt tolerance in rice (Oryza sativa L.). Omonrice, 16: 50-56 (2008) 
Lin, M.H.; C.W. Lin; J.C. Chen; Y.C. Lin; S.Y. Cheng; T.H. Liu; F.J. Jan; S.T. Wu; F.S. Thseng and H.M. Ku(2007). Tagging rice droughtrelated QTL with SSR DNA markers. Crop, Environment and Bioinformatics, 4: 65-76.

Lodha, T.; J. Karmakar; R. Roychoudhuri and N. Dey(2011). Assessment of genetic diversity of some commonly grown rice genotypes of South Bengal using microsatellite markers associated with the saltol QTL mapped on 1st chromosome. NBU J. Plant Sci, 5:35-39

Massonnet, E.C.; R. Serge; D. Erwin and J.L. Regnard(2007). Stomatal regulation of photosynthesis in apple leaves: Evidence for different water-use strategies between two cultivars Catherine. Ann. Bot., 100(6): 1347-1356.

Matin, S.; M. Ashrafuzzaman; M.M. Islam; S.U. Sikdar and N. Zobayer(2012). Molecular marker based (SSR) genetic diversity analysis in deep water rice germplasms of Bangladesh. International Journal of Biosciences (IJB) ISSN: 2222-5234 Vol. 2, No. 10(2), p. 64-72, 2012.

Mohammadi-Nejad, G.; A. Arzani; A.M. Rezaie; R.K. Singh and G.B. Gregorio(2008). Assessment of rice genotypes for salt tolerance using microsatellite markers associated with the saltol QTL. African Journal of Biotechnology Vol. 7 (6), pp. 730-736, 18 March, 2008.

Murray, A.A. and W.F. Thompson(1980). Rapid isolation of high molecular weight plant DNA. Nucleic Acid Research, 8: 4321-4325.

Nagy, S.; P. Poczai; I. Cernak; A.M. Gorji; G. Hegedus and J. Taller(2012). PICcalc: An Online Program to Calculate Polymorphic Information Content for Molecular Genetic Studies. Biochemical Genetics, 50: 670-672.

Nei, M.(1973). Analysis of gene diversity in subdivided populations. Proceeding of the National Academy of Sciences of the United States of America, 70(12) Part 1: 3321-3323.

Ni, J.; P.M. Colowit and D.J. Mackill(2002). Evaluation of genetic diversity in rice subspecies using microsatellite markers. Crop Science, 42: 601-607.

Ram, S.G.; V. Thiruvengadam and K.K. Vinod(2007). Genetic diversity among cultivars, landraces and wild relatives of rice as revealed by microsatellite markers. Journal of Applied Genetics, 48(4): 337345.

Ramadan, E.A.; A.M. Elmoghazy and H.F. El-Mowafi(2015). Molecular Markers based Genetic Diversity Analysis for Drought Tolerance in Rice (Oryza Sativa, L.) Using SSR Markers. International Journal of Scientific Research in Agricultural Sciences, 2(Proceedings), pp. 137-146, 2015.

Richards, L.A.(1954). Diagnosis and Improvement of Saline and Alkaline Soils.United States Salinity Laboratory Staff. Agricultural Handbook No 60. United States Department of Agriculture, 160p. 
RRTC (2011). Rice Research and Training Center. Annual rice national campaign report of rice program, Agric. Res. Cen., Ministry of Agriculture and Land Reclamation.

RRTC (2014). Technology transfer report of rice program, Rice Research and Training Center., Agric. Res. Cen., Ministry of Agriculture and Land Reclamation.

Sadasivam, S. and A. Manickam(1996). Biochemical Methods, New Age International Publishers (P) Ltd., New Delhi, India.

Sajib, A.M.; M.M. Hossain; A.T.M. Mosnaz; H. Hossain; M.M. Islam; M.S. Ali and S.H. Prodhan(2012). SSR marker-based molecular traitization and genetic diversity analysis of aromatic landreces of rice (Oryza sativa, L.). Journal of Bio-Science and Biotechnology, 1(2): 107-116.

Sedeek, S.E.M.; R.A. El-Namaky; S.A.A. Hammoud and Howida B. ELHabet(2012). Genetical studies on root system and yield and its components traits under water limit condition in rice (Oryza sativa I.) j.agric.chem.and biotechn., mansoura univ.vol. 3 (12): 447 - 460, 2012.

Shehata, S.M.; R.A. EI-Namaky.; S.E. Sedeek and E.A. Badr(2009). Gene action and combining ability of quantitative traits and related criteria of disease (blast \&brown spot) resistance under saline soil condition in rice. Egypt. J. of Appl. Sci., 24(10): 186-204.

Snedecor, G.W. and W.G. Cochran(1967). Statistical methods. 6th ed. lowa State Univ. Press, Ames, lowa, U.S.A.

USDA, (2013). Bibliography on Salt Tolerance. Fibres, Grains and Special Crops. Riverside, CA: George E. Brown, Jr. Salinity Lab.US Department Agriculture, Agriculture Research Service.

Vanniarajan, C.; K.K. Vinod and A. Pereira(2012). Molecular evaluation of genetic diversity and association studies in rice (Oryza sativa, L.). Journal of Genetics, 91(1): 1-11.

Weerakoon, W.M.W.; A. Maruyama and K. Ohba(2008). Impact of humidity on temperature induced grain sterility in rice (Oryza sativa L.). Rice Research and Development Institute, Bata lagoda, Ibba gamuwa, Sri Lanka; Journal of Agronomy and Crop Science, 194(2): 135-140.

Weir, B.S.(1989). Sampling properties of gene diversity. In: Brownp, A. H. D.; M. T. Clegg; H. L. Kahler and B. S. Weir (eds) plant population genetics, breeding and genetic resources. Sinauer Associates, Sunderland, Massachusetts, 23-42 pp.

Welsh, J. and M. McClelland(1990). Fingerprinting genomes using PCR with arbitrary primers. Nucleic Acids Res., 18: 7213-7218

Witcombe, J.R.; P.R. Hollington; C.J. Howarth; S. Reader; and K.A. Steele(2008). Breeding for abiotic stresses for sustainable agriculture. Philosophical Transactions of The Royal Society Biology 363, 703-716.

Zayed, B.A.; K.S. Abd El-azeem and A.M.A Osama(2014). Physiological characterization of Egyptian salt tolerantrice varieties under 
different salinity levels. Life Science Journal 2014;11(10).12641272.

Zeng, L., T.R. Kwon; X. Liu; C. Wilson; C.M. Grieve and G.B. Gregorio(2004). Genetic diversity analyzed by microsatellite markers among rice (Oryza sativa, L.) genotypes with different adaptations to saline soils. Plant Science, 166: 1275-1285.

Zhou, J.; X. Wang; Y. Jiao; Y. Qin; X. Liu; K. He; C. Chen; L. Ma; J. Wang; L. Xiong; Q. Zhang; L. Fan; and X.W. Deng(2007). Global genome expression analysis of rice in response to drought and highsalinity stresses in shoot, flag leaf, and panicle. Plant Mol Biol. 63: 591-608.

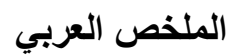

$$
\begin{aligned}
& \text { دراسات وراثية وجزيئية على تحمل الملوحة والجفاف فى الأرز } \\
& \text { منى على فريد إبراهيم*، على أحمد أبو شوشة*، محروس السبد عبد الباقى ** وسعيد محمد } \\
& \text { * قسم الوراثه ـ كلية الزراعة ـ جامعة كفر الثيخ. ** مركز البحوث والتدريب فى الارز سمركز }
\end{aligned}
$$

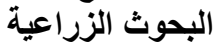

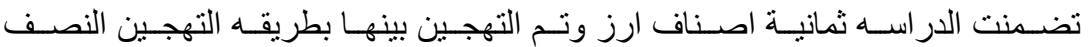

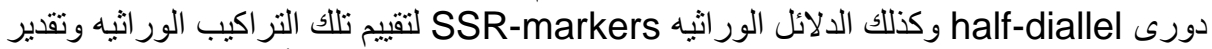

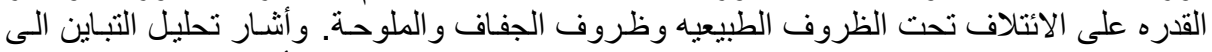

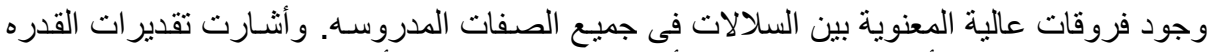

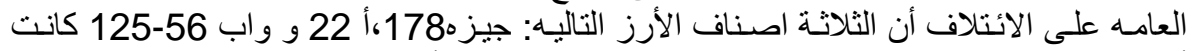

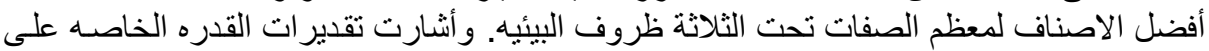

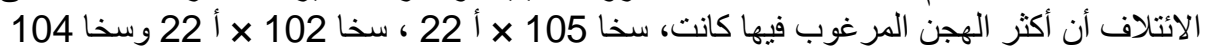

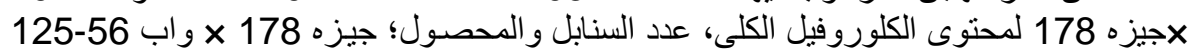

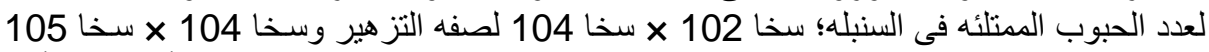

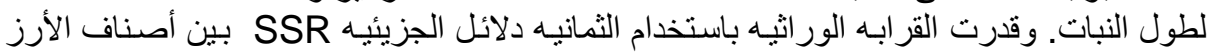

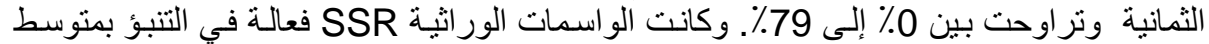

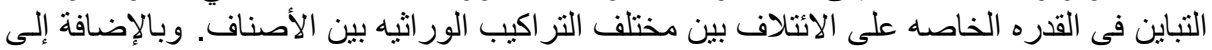

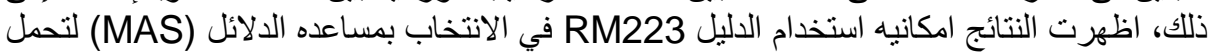

$$
\begin{aligned}
& \text { الملوحة و الجفاف، النهات. }
\end{aligned}
$$

